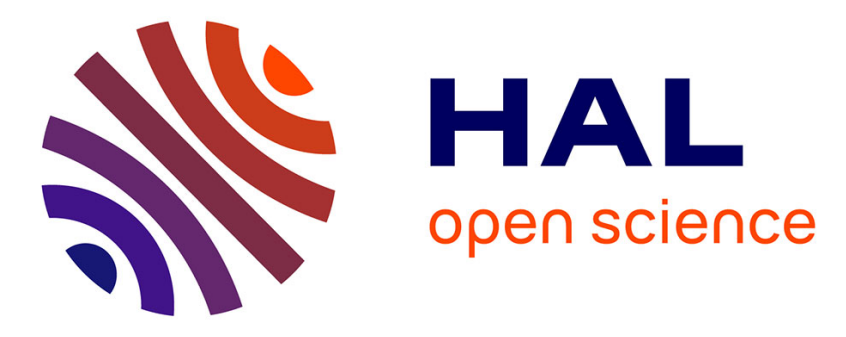

\title{
Libration-driven flows in ellipsoidal shells
}

D. Lemasquerier, A. M. Grannan, J. Vidal, D. Cebron, B. Favier, M. Le Bars, J. M. Aurnou

\section{To cite this version:}

D. Lemasquerier, A. M. Grannan, J. Vidal, D. Cebron, B. Favier, et al.. Libration-driven flows in ellipsoidal shells. Journal of Geophysical Research. Planets, 2017, 122 (9), pp.1926-1950. 10.1002/2017JE005340 . hal-01764691

\section{HAL Id: hal-01764691 https://hal-amu.archives-ouvertes.fr/hal-01764691}

Submitted on 3 Jan 2022

HAL is a multi-disciplinary open access archive for the deposit and dissemination of scientific research documents, whether they are published or not. The documents may come from teaching and research institutions in France or abroad, or from public or private research centers.
L'archive ouverte pluridisciplinaire HAL, est destinée au dépôt et à la diffusion de documents scientifiques de niveau recherche, publiés ou non, émanant des établissements d'enseignement et de recherche français ou étrangers, des laboratoires publics ou privés. 


\section{Journal of Geophysical Research: Planets}

\section{RESEARCH ARTICLE \\ Libration-driven flows in ellipsoidal shells}

10.1002/2017JE005340

\author{
Key Points: \\ - Libration-driven elliptical \\ instability (LDEI) persists in the more \\ geophysically relevant ellipsoidal \\ shell geometry \\ - In ellipsoidal shell geometries, LDEI \\ differs between the regions inside \\ and outside the inner core tangent \\ cylinder \\ - LDEl is capable of driving space-filling \\ turbulence in planetary cores \\ (e.g., $55 \mathrm{CnC}$ e) and subsurface oceans \\ (e.g., Enceladus)
}

Supporting Information:

- Table S1

- Supporting Information S1

Correspondence to:

D. Lemasquerier,

d.lemasquerier@gmail.com

\section{Citation:}

Lemasquerier, D., A. M. Grannan, J. Vidal, D. Cébron, B. Favier, M. Le Bars, and J. M. Aurnou (2017), Libration-driven flows in ellipsoidal shells, J. Geophys. Res. Planets Planets, 122, 1926-1950, doi:10.1002/2017JE005340.

Received 8 MAY 2017 Accepted 17 AUG 2017 Accepted article online 24 AUG 2017 Published online 21 SEP 2017

○2017. American Geophysical Union. All Rights Reserved.

\section{Introduction}

\subsection{Context}

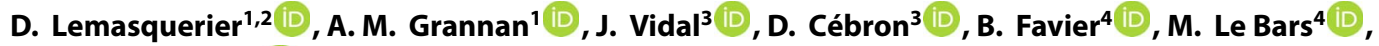 \\ and J. M. Aurnou' 1 (D) \\ ${ }^{1}$ Department of Earth, Planetary, and Space Sciences, University of California, Los Angeles, California, USA, ${ }^{2}$ Département \\ des Sciences de la Terre, École Normale Supérieure de Lyon, Université de Lyon, Lyon, France, ${ }^{3}$ CNRS, ISTerre, Université \\ Grenoble Alpes, Grenoble, France, ${ }^{4}$ CNRS, Aix Marseille University, Centrale Marseille, IRPHE, Marseille, France
}

\begin{abstract}
Planets and satellites can undergo physical librations, which consist of forced periodic variations in their rotation rate induced by gravitational interactions with nearby bodies. This mechanical forcing may drive turbulence in interior fluid layers such as subsurface oceans and metallic liquid cores through a libration-driven elliptical instability (LDEI) that refers to the resonance of two inertial modes with the libration-induced base flow. LDEl has been studied in the case of a full ellipsoid. Here we address for the first time the question of the persistence of LDEl in the more geophysically relevant ellipsoidal shell geometries. In the experimental setup, an ellipsoidal container with spherical inner cores of different sizes is filled with water. Direct side view flow visualizations are made in the librating frame using Kalliroscope particles. A Fourier analysis of the light intensity fluctuations extracted from recorded movies shows that the presence of an inner core leads to spatial heterogeneities but does not prevent LDEI. Particle image velocimetry and direct numerical simulations are performed on selected cases to confirm our results. Additionally, our survey at a fixed forcing frequency and variable rotation period (i.e., variable Ekman number, $E$ ) shows that the libration amplitude at the instability threshold varies as $\sim E^{0.65}$. This scaling is explained by a competition between surface and bulk dissipation. When extrapolating to planetary interior conditions, this leads to the $E^{1 / 2}$ scaling commonly considered. We argue that Enceladus' subsurface ocean and the core of the exoplanet $55 \mathrm{CnC}$ e should both be unstable to LDEl.
\end{abstract}

Plain Language Summary Because of their gravitational interactions with other bodies, planets and moons are subjected to mechanical forcings that perturb their spin rate. The motivation of this study is to determine whether one of these forcings, called libration, can drive global-scale flows in interior fluid layers, like the subsurface ocean of Europa or the liquid inner core of lo. Turbulent flows in these layers are of interest because they can be linked to the generation of magnetic fields, planetary heat fluxes, and energy dissipation rates. Furthermore, since it has been proposed that life may be harbored within these subsurface oceans, their internal structure and dynamics are of broad interest to the planetary science community and beyond. To model libration experimentally, containers of a given geometry are filled with water and are made to librate. Previous studies have shown that the flow can become unstable for precise oscillation frequencies. By combining laboratory experiments, numerical simulations, and a theoretical analysis, we show for the first time that this instability persists in an ellipsoidal shell geometry, i.e., an ellipsoid inside of which is suspended a spherical inner core. This result is of primary importance since most liquid cores and subsurface oceans are expected to have this geometry. Furthermore, our results show that the generated turbulence can be latitudinally inhomogeneous. By performing a survey, we extrapolate our results to planetary interior conditions and show that libration is capable of driving turbulence in planetary cores (e.g., the exoplanet $55 \mathrm{CnC}$ e) and subsurface oceans (e.g., Enceladus).

Planets and moons spin around their rotation axis at a given angular velocity. However, they are subjected to several types of mechanical forcings that periodically perturb this rotation, such as precession and libration. This study focuses on longitudinal libration, which physically corresponds to an oscillation of the axial rotation rate of a body that results from gravitational interactions with an orbital partner.

The effects of these mechanical forcings on the dynamics of internal fluids are of major interest for planetary bodies that have a liquid metal core (e.g., Mercury, the Moon, lo, and Ganymede) and for bodies that have 
subsurface oceans (e.g., Europa, Callisto, Ganymede, Enceladus, and Titan). In these bodies, internal flows are linked to the generation of magnetic field, planetary heat fluxes, and energy dissipation. A better understanding of these flows is thus important to consider relevant hydrodynamical effects in modelings. Furthermore, since it has been proposed that life may be harbored within these subsurface oceans, their internal structure and dynamics are of broad interest to the planetary science community and beyond. The motivation here is thus to determine whether mechanical forcing can drive strong global-scale flows by injecting energy into interior fluid layers.

One way to perform this exchange of energy is through a viscous coupling between the solid and liquid layers. That is why the first studies of the fluid dynamical effects of libration focused on spherical geometry (full sphere and spherical shell). In such geometries, longitudinal libration excites inertial waves, which find their origin in the restoring effect of the Coriolis force. For a finite fluid volume, inertial waves are reflected by the solid walls. These waves can then combine through constructive interference to form global inertial modes [Greenspan, 1968; Aldridge and Toomre, 1969; Rieutord, 1991; Noir et al., 2009]. However, no resonant response is expected in the limit of small Ekman number and forcing amplitude [Zhang et al., 2013].

When an elliptically deformed body undergoes longitudinal libration [e.g., Cébron et al., 2012a; Noir et al., 2012; Grannan et al., 2014; Favier et al., 2015], a topographic torque is generated between the solid outer boundary (e.g., the mantle) and the interior fluid layer (e.g., the liquid core). This mechanical forcing can excite a parametric fluid instability involving two inertial modes of the rotating flow plus the elliptically deformed basic flow in response to the harmonic forcing [Le Bars et al., 2015]. This instability is called the libration-driven elliptical instability (LDEI).

\subsection{Motivations}

It is currently unclear whether the LDEl investigated in the full ellipsoidal cavity can be extended to the more geophysically relevant shell geometry. While the solutions for inertial modes have been calculated for a full cylinder and cylindrical shells [Herreman et al., 2009], as well as in spheroidal [Zhang et al., 2004] and ellipsoidal [Vantieghem, 2014] cavities, the complete spectrum of eigenfrequencies is unknown for spherical or ellipsoidal shells. In such a configuration, and for very weak libration forcing, the only known regular inviscid solutions are purely toroidal modes because of the new constraints imposed by the inner boundary [Rieutord et al., 2001]. Inertial modes are instead confined along singular paths of characteristics and form the so-called attractors. When adding viscosity but remaining in a regime where the Coriolis force largely dominates the viscous force (typically Ekman number $\sim 10^{-8}$ ), the singularities take the form of thin shear layers localized around the inviscid attractors [Rieutord and Valdettaro, 2010]. This suggests that LDEI might be significantly modified in ellipsoidal shells since inertial modes are not expected to robustly and globally develop in such geometries. Previous studies of the elliptical instability in shell geometries have been conducted [Seyed-Mahmoud et al., 2000, 2004; Lacaze et al., 2005; Cébron et al., 2010b]. These studies focus on the case of a tidal forcing rather than libration and do not explore the developed turbulent regime following the elliptical instability. The goal of the present investigation is to experimentally and numerically demonstrate that inertial mode resonances do indeed develop in librating ellipsoidal shells and thus that LDEI can exist in the subsurface oceans and liquid metal cores of librating bodies.

\section{Theoretical Formalism}

\subsection{Model and Equations}

We consider the flow $\mathbf{u}$ of an incompressible $(\nabla \cdot \mathbf{u}=0)$ fluid of uniform density and kinematic viscosity $v$. The fluid is enclosed between a rigid ellipsoidal outer container, whose surface, in a reference frame fixed to the surface, is defined in Cartesian coordinates by

$$
\frac{x^{2}}{a^{2}}+\frac{y^{2}}{b^{2}}+\frac{z^{2}}{c^{2}}=1
$$

where $a, b$, and $c$ are the long equatorial axis, the short equatorial axis, and the polar axis, respectively. We define the mean external radius as $R=\sqrt{\left(a^{2}+b^{2}+c^{2}\right) / 3}$ and the equatorial ellipticity as

$$
\beta=\frac{a^{2}-b^{2}}{a^{2}+b^{2}} .
$$

The aspect ratio of the shell is $\chi=r_{i} / R$ where $r_{i}$ is the radius of the spherical inner core. The container (outer ellipsoid and inner core) is subjected to longitudinal libration, i.e., rotates around the vertical axis $\hat{\mathbf{z}}$ with 
a time-dependent spin rate given by $\Omega_{\text {spin }}(t)=\Omega_{0}+\Delta \phi \omega_{\text {lib }} \sin \left(\omega_{\text {lib }} t\right)$, where $\Omega_{0}$ is the mean spin rate, $\Delta \phi$ is the libration amplitude, and $\omega_{\text {lib }}$ is the angular libration frequency.

We work in the body frame attached with the walls of the librating container (librating frame). The inner and outer boundaries are fixed in that frame. We choose $\Omega_{0}^{-1}$ as the time scale and the shell thickness $R(1-\chi)$ as the length scale. The dimensionless spin rate is

$$
\Omega(t)=1+\epsilon \sin (f t)
$$

with $\epsilon=f \Delta \phi$ the dimensionless libration amplitude and $f=\omega_{\text {lib }} / \Omega_{0}$ the dimensionless libration frequency. In the librating frame the momentum and continuity equations for the velocity field $\mathbf{u}$ are

$$
\begin{gathered}
\frac{\partial \mathbf{u}}{\partial t}+\mathbf{u} \cdot \nabla \mathbf{u}+2 \boldsymbol{\Omega} \times \mathbf{u}=-\nabla \Pi+E_{\chi} \nabla^{2} \mathbf{u}-\underbrace{\frac{\mathrm{d} \boldsymbol{\Omega}}{\mathrm{dt}} \times \mathbf{r}}_{\text {Poincarè }} \\
\nabla \cdot \mathbf{u}=0,
\end{gathered}
$$

with $E_{\chi}=v /\left[\Omega_{0} R^{2}(1-\chi)^{2}\right]$ the Ekman number (dimensionless viscosity), $\Pi$ the reduced pressure taking into account the centrifugal acceleration, and $\mathbf{r}$ the dimensionless position vector. In equation (4) the last term on the right-hand side is the Poincare force generated by the nonuniform rotation of the librating frame. Finally, the velocity field satisfies the no-slip boundary condition $\mathbf{u}=\mathbf{0}$ at both the inner spherical and outer ellipsoidal surfaces.

\subsection{Basic Flow}

We consider first the equatorial plane $(z=0)$ of our system to determine the two-dimensional base flow $\mathbf{U}$ bounded by an external elliptical boundary and an inner circular boundary. We work in cylindrical coordinates $(s, \phi, z)$ in the librating frame. The flow is described by the stream function $\psi$ such that $\mathbf{U}=\nabla \times[-\epsilon \sin (f t) \psi \hat{\mathbf{z}}]$. In the inviscid limit $E_{\chi}=0$, the flow satisfies the nonpenetration conditions $u_{s}=0$ at the inner and outer boundaries. Assuming a small equatorial ellipticity $\beta \ll 1$, we expand the stream function as $\psi=\psi_{0}+\beta \psi_{1}$ where $\psi_{0}=\left(s^{2}-1\right) / 2$ is the stream function of the solid-body rotation and $\psi_{1}$ is the first-order elliptical correction. With the ansatz $\psi_{1}=F(s) \cos (2 \phi) / 2$, the inviscid vorticity equation reduces to the Laplace equation $\nabla^{2} F=0$, yielding

$$
\psi_{1}=\left(\frac{A_{1}}{s^{2}}+B_{1} s^{2}\right) \frac{\beta}{2} \cos (2 \phi)
$$

where $A_{1}=-\chi^{4} /\left(1-\chi^{4}\right)$ and $B_{1}=1 /\left(1-\chi^{4}\right)$ are fixed by the boundary conditions. The complete stream function is thus

$$
\psi=\frac{s^{2}-1}{2}+\left(\frac{A_{1}}{s^{2}}+B_{1} s^{2}\right) \frac{\beta}{2} \cos (2 \phi)
$$

and the cylindrical flow components are reduced to

$$
\begin{gathered}
U_{s}=\epsilon \sin (f t)\left(\frac{A_{1}}{s^{3}}+B_{1} s\right) \beta \sin (2 \phi), \\
U_{\phi}=\epsilon \sin (f t)\left[s+\left(-\frac{A_{1}}{s^{3}}+B_{1} s\right) \beta \cos (2 \phi)\right] .
\end{gathered}
$$

In the librating frame, each fluid parcel thus oscillates back and forth along a part of an elliptical streamline whose flattening depends on the distance from the inner core boundary.

In our experimental setup the basic flow $\mathbf{U}$ is a priori three-dimensional because of the viscous corrections. However, for $z \in[-\chi, \chi]$, the base flow is enclosed between an ellipsoidal outer boundary and a spherical inner core. We therefore neglect the vertical component and approximate the base flow $\mathbf{U}$ by the horizontal components (8) and (9). We thus compute the basic flow as a purely two-dimensional flow, defined for each $z$ position by replacing $\chi$ with $\sqrt{\chi^{2}-z^{2}}$ to calculate $A_{1}$ and $B_{1}$. For $|z|>\chi$, the base flow is enclosed within an ellipsoidal boundary without any inner boundary, and the horizontal base flow reduces to (8) and (9) with $A_{1}=0$ and $B_{1}=1$. 


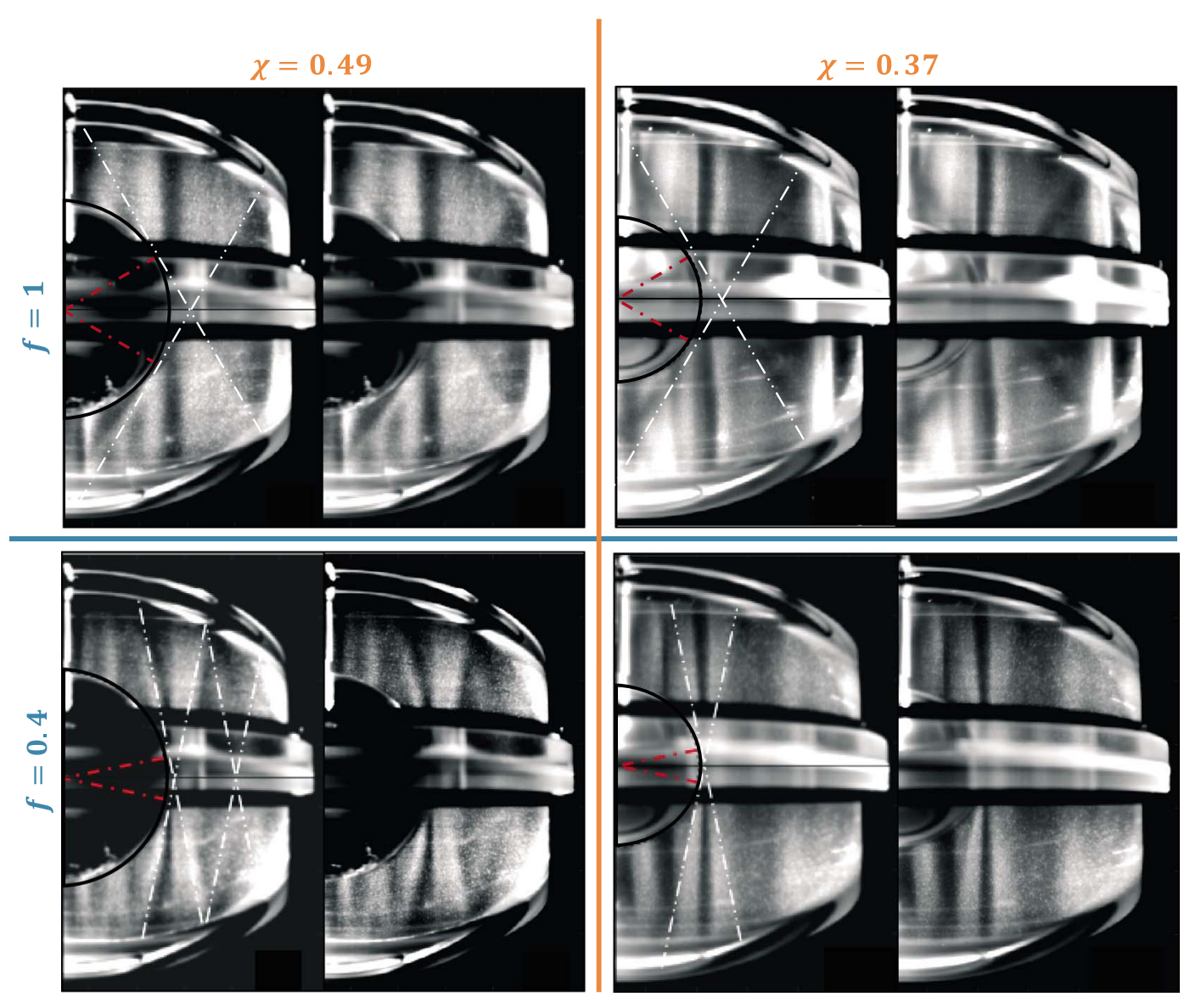

Figure 1. Kalliroscope visualizations of the shear layers formed by the direct excitation of inertial waves for two different forcing frequencies ( $f=1$ and 0.4 ) and two different core sizes ( $\chi=0.49$ and 0.37 ). Each image is obtained by stacking instantaneous snapshots extracted at $t=n T_{\text {lib }}+T_{\text {lib }} / 2$, where $T_{\text {lib }}$ is the libration period. From left to right and top to bottom, the stacking is performed over 50,50, 45, and 41 libration periods. Two images are given for each parameter set: a raw image on the right and the same image on the left where we have superimposed dashed white lines representing the theoretical direction of the shear layers $(\theta=\arccos (f / 2))$ and dashed red lines pointing toward the critical latitude $\left(\alpha_{c}=\arcsin (f / 2)\right)$.

\subsection{Inertial Waves}

A rotating fluid in an unbounded medium supports oscillatory motions called inertial waves. The latter are solutions of [Greenspan, 1968]

$$
\begin{gathered}
\frac{\partial \mathbf{u}}{\partial t}+2 \hat{\mathbf{z}} \times \mathbf{u}=-\nabla \Pi, \\
\nabla \cdot \mathbf{u}=0,
\end{gathered}
$$

which can be rearranged to give a single equation for the pressure field (the Poincaré equation). Equation (10) admits plane wave solutions $\mathbf{u} \propto e^{i(\mathbf{k} \cdot \mathbf{r}+\omega t)}$, where $\mathbf{k}$ is the dimensionless wave vector and $\omega$ is the dimensionless frequency. These inertial waves satisfy the dispersion relation

$$
\omega= \pm 2 \cos \theta
$$

where $\theta$ is the angle between $\mathbf{k}$ and the axis of rotation $\widehat{\mathbf{z}}$. The dispersion relation in (12) shows that $|\omega|<2$ and that inertial waves are dispersive and anisotropic. For a finite fluid volume, inertial waves reflect on solid walls, keeping $\theta$ constant according to (12) and can generate global inertial modes through constructive interference.

Experimental [Aldridge and Toomre, 1969; Noir et al., 2009] and numerical [Rieutord, 1991; Tilgner, 1999; Calkins et al., 2010] studies show that longitudinal libration can excite inertial modes, although a direct resonance mechanism is not predicted by theoretical studies [Zhang et al., 2011, 2013]. In viscous spherical shells, 
internal shear layers, i.e., superposition of inertial waves [Kerswell, 1995], are spawned from the so-called critical latitude where the energy of incoming inertial waves is reflected along the boundary [Phillips, 1963]. Shear layers are also associated with the breakdown of the Ekman boundary layer [Greenspan, 1968]. For a given forcing frequency $f$, the critical latitude $\alpha_{c}$ is determined by $f=2 \sin \alpha_{c}$.

Since inertial modes are the starting point for the stability analysis of libration, precession, or tidally driven flows, their investigation is of interest. In Figure 1 we show direct excitations of inertial waves using Kalliroscope visualizations for forcing frequencies $f \leq 2$. The shear layers are qualitatively observed at critical colatitudes in good agreement with the theoretical predictions. However, the precise study of direct resonance of inertial waves in ellipsoidal shells is beyond the scope of this study.

\subsection{Elliptical Instability}

The libration-driven elliptical instability (LDEI) is a linear instability mechanism that arises from the resonant interaction of triads of waves, namely, two inertial waves plus the elliptical deformation of the fluid streamlines by the oscillating boundaries [Cébron et al., 2012b; Vidal et al., 2017]. Expanding velocity and pressure perturbations around the basic state as a linear combination of inertial modes, one can show that the LDEI grows in time if the following resonance conditions are satisfied [Grannan et al., 2014]:

$$
\begin{gathered}
\left|m_{1}-m_{2}\right|=m_{\text {lib }}=2, \\
\left|\omega_{1}-\omega_{2}\right|=f,
\end{gathered}
$$

where $m_{i}$ is the azimuthal wave number and $\omega_{i}$ is the eigenfrequency of the $i$ th inertial mode of the triad. The azimuthal wave number $m_{\text {lib }}=2$ of the base flow $\mathbf{U}$ is a direct consequence of the fact that the outer surface of the container is ellipsoidal. Because of the dispersion relation (12), $\left|\omega_{i}\right| \leq 2$. In the asymptotic limit of $\beta, \epsilon \rightarrow 0$ (i.e., weak ellipticity and weak libration amplitude, relevant for planets and moons), the elliptical instability exists only if $|f|<4$, whereas finite values of $\beta$ and $\epsilon$ allow instabilities when $|f|<4+\epsilon \beta$ (see Grannan et al. [2014] for details).

In this study, we realize a survey with a fixed frequency $f=4$ for several reasons. First, in this frequency regime, no inertial waves are directly excited by the forcing. It allows us to focus only on the LDEI mechanism, i.e., on an indirect excitation of inertial modes. Second, this case is the one where the determination of the modes and frequency coupling is the simplest. Inertial modes with eigenfrequencies $f_{1,2} \simeq f / 2=2$ meet the resonance conditions in (14) and are particularly easy to identify with a side view visualization. Indeed, $\mathbf{k}$ is, in this case, parallel to the rotation axis, implying that the group velocity of the excited waves is horizontal. These were identified as the $\Lambda_{8, \pm 1,7}$ modes in the absence of inner core [Grannan et al., 2014; Favier et al., 2015] using the description of inertial modes in a rotating spheroid given by Kerswell [1994]. Since the radial component of this mode is not zero, by definition, it is not a purely toroidal mode. It is thus also a way to verify if modes having a poloidal component can be excited in an ellipsoidal shell. Additional cases have also been done with the forcing frequency $f=2.4$ to show that the spin-over mode (solid-body rotation inclined with the rotation axis) is still excited.

\subsection{Local Stability Analysis}

Cébron et al. [2012b, 2014] performed the local stability analysis of libration-driven basic flows valid in full ellipsoids. The local stability method probes the stability of the pathlines of the basic flow, considering inviscid plane wave perturbations of small wavelengths [Le Dizès, 2000]. The local inviscid growth rate $\sigma_{\text {inv }}$ of LDEl is at first order in $\epsilon \beta$ [Cébron et al., 2012b]

$$
\sigma_{\text {inv }}=\frac{16+f^{2}}{64} \epsilon \beta
$$

Using the same approach, the inviscid growth rate of LDEI upon the libration-driven base flow in (8) and (9) is

$$
\sigma_{\text {inv }}^{\prime}=\frac{\left(16+f^{2}\right)\left|3 A_{1}+B_{1} s^{4}\right|}{64 s^{4}} \epsilon \beta
$$

The growth rate for the full ellipsoid in (15) is recovered from (16) when $A_{1}=0$ and $B_{1}=1$. Note that because the streamline deformation is changing with $s$ and $z$, the growth rate $(16)$ is spatially varying. However, for $\chi \in[0,0.74]$, the spatial mean (along s) of $\sigma_{\text {inv }}^{\prime}$ is always smaller than $\sigma_{\text {inv. }}$. The highest growth rate, given by (15), is the one used in this study. This choice is later supported by the fact that the instability is seen to grow primarily close to the poles where $A_{1}=0$ and $B_{1}=1$. 
To include dissipative terms due to the no-slip boundary conditions, Cébron et al. [2012b] assumed that dissipation mainly occurs in the Ekman boundary layer of thickness $E_{\chi}^{1 / 2}$. The viscously damped growth rate of LDEl is then

$$
\sigma=\sigma_{\text {inv }}-\alpha \Psi(\chi) \sqrt{E_{\chi}},
$$

where $\alpha$ is a constant of order unity and $\Psi$ a function taking into account the dependence of the damping with the radius ratio of the shell $\chi$. Hollerbach and Kerswell [1995] show that the tilt-over mode, corresponding to the basic flow of a precessing shell, is damped viscously following $\Psi=(1-\chi)\left(1+\chi^{4}\right) /\left(1-\chi^{5}\right)$. This tilt-over mode is similar to the so-called spin-over mode of the TDEl (tidally driven elliptical instability). No generic formula exists to quantify $\Psi$ for other modes of the elliptical instability excited by tides or libration.

\section{Methods}

\subsection{Experimental Setup}

\subsubsection{Description of the Experiment}

The container used is a polished acrylic cavity made from two nonaxisymmetric hemispheres. The fluid cavity dimensions are $a=12.7 \mathrm{~cm}$ and $b=c=8.9 \mathrm{~cm}$, which gives an equatorial ellipticity of $\beta=0.34$. A solid acrylic inner core is added inside the ellipsoidal cavity using a metallic rod suspended from the top of the acrylic container. The radius of the inner cores used are $r_{i}=[2.51,3.82,5.07,6.12,7.62] \mathrm{cm}$ corresponding to $\chi=[0.24,0.37,0.49,0.59,0.74]$.

This container is fixed on the same device as the one used previously by Noir et al. [2009, 2010, 2012] and Grannan et al. [2014]. Two motors are used to replicate a librational forcing. The first one rotates a $1 \mathrm{~m}$ diameter turntable at a constant rotation rate $\Omega_{0}$ varying from 1 to $60 \mathrm{rpm}(0.017 \mathrm{to} 1 \mathrm{~Hz})$. The second one, which is mounted on this turntable, is directly coupled to the acrylic cavity and superimposes a sinusoidal oscillation $\Delta \phi \omega_{\text {lib }} \sin \left(\omega_{\text {lib }} t\right)$ (see Figure 2). In this study, the container oscillations are characterized by an amplitude $2 \Delta \phi \in\left[0^{\circ}, 65^{\circ}\right]$ and a frequency $\omega_{\text {lib }} / 2 \pi \in[0,3.84 \mathrm{~Hz}]$. Top-facing and side-facing cameras, shown in Figure 2 , are used to perform visualizations described in section 3.2.

\subsubsection{Cases Realized}

Figure 3 shows the Ekman number as a function of the background rotation rate $\Omega_{0}$, using colored curves for the six different values of $\chi$. The upper $x$ axis shows the dimensional frequency of libration for a fixed nondimensional frequency $f=4$. The horizontal dash-dotted lines show the six Ekman numbers used in the experiments, and the black dots show the intersection of these fixed Ekman values with the solid curves.

Each experiment is conducted the same way. A constant rotation is applied for several minutes until the fluid reaches solid-body rotation. The two cameras start recording movies simultaneously, and the oscillation of the acrylic container is then activated. For each set of parameters $\left(E_{\chi}, \chi\right)$ (black dots in Figure 3$), \Delta \phi$ is adjusted to determine an approximate amplitude threshold for the instability. To determine whether a case is stable or unstable, we wait for five predicted growth times using (17) with $\Psi \approx(1-\chi)$ and visually check whether a turbulent flow develops or not. For some unstable cases, we record longer movies ( $\sim 10 \mathrm{~min})$ to be able to perform a signal analysis on both the growing and the fully turbulent phases. Table 1 recapitulates the experimental parameters, definitions, and ranges explored. A complete table of all the cases realized is available in the supporting information file (Table S1).

\subsection{Flow Analysis Methods}

\subsubsection{Kalliroscope and Particle Image Velocimetry}

Direct side view visualizations of the flow are performed by seeding the water with Kalliroscope particles and illuminating the tank with a meridional laser plane. These particles are thin plates that reflect light preferentially along the direction of their short axis and orient themselves with the shear of the flow. Their collective reflectance thus gives a visual indication of their orientation and, thereby, of the flow behavior [Hecht et al., 2010]. The two lasers used to create the light sheet are attached to the librating frame. Two cameras are used to acquire $1920 \times 1280$ resolution movies of the flow at 30 frames per second. A GoPro Hero4 Silver camera is fixed in the librating frame and acquires movies in the narrow mode to avoid optical distortion. The second camera is a Canon EOS 7D digital camera fixed in the rotating frame. The GoPro angle of view focuses on one quadrant of the cavity, whereas the Canon EOS 7D allows to visualize the whole shell.

To evaluate the information obtained from Kalliroscope visualizations, a selected case is chosen and analyzed using both Kalliroscope particles and particle image velocimetry (PIV) in a vertical plane and in a horizontal 
(a)

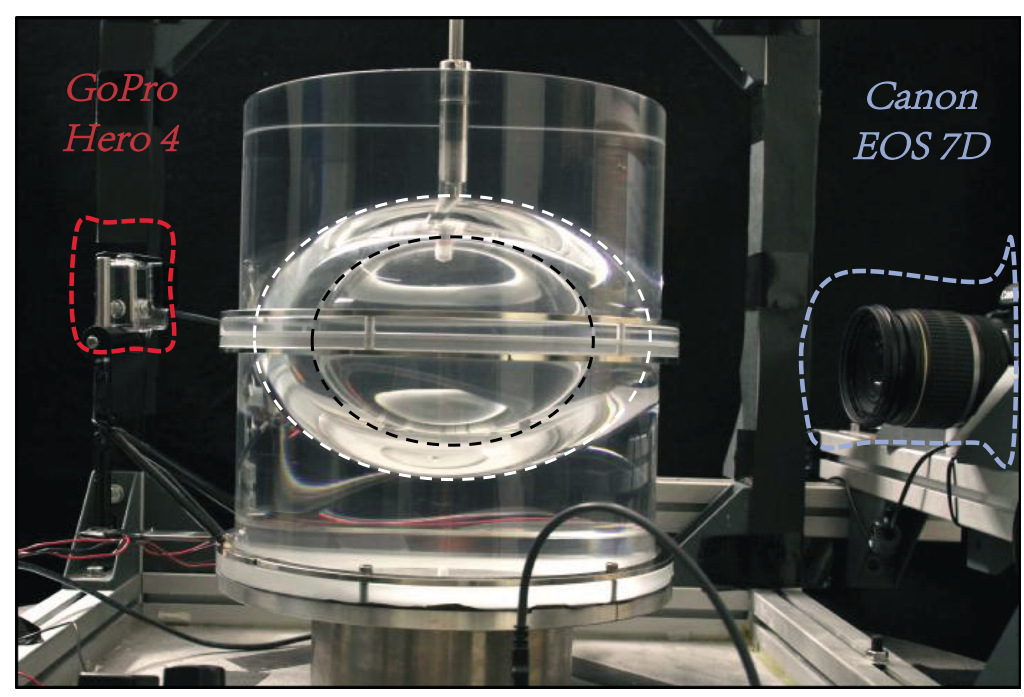

(b)
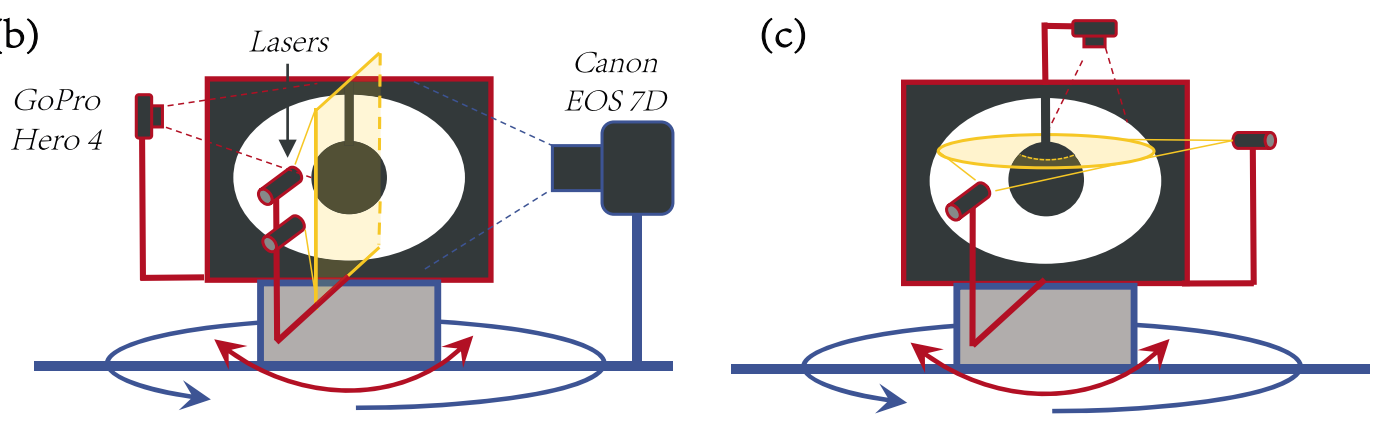

Figure 2. (a) Side view image and (b, c) schematic representations of the laboratory experiment used to perform visualizations on vertical and horizontal planes. The inner core is spherical, even if it appears ellipsoidal in Figure 2a due to optical distortions.

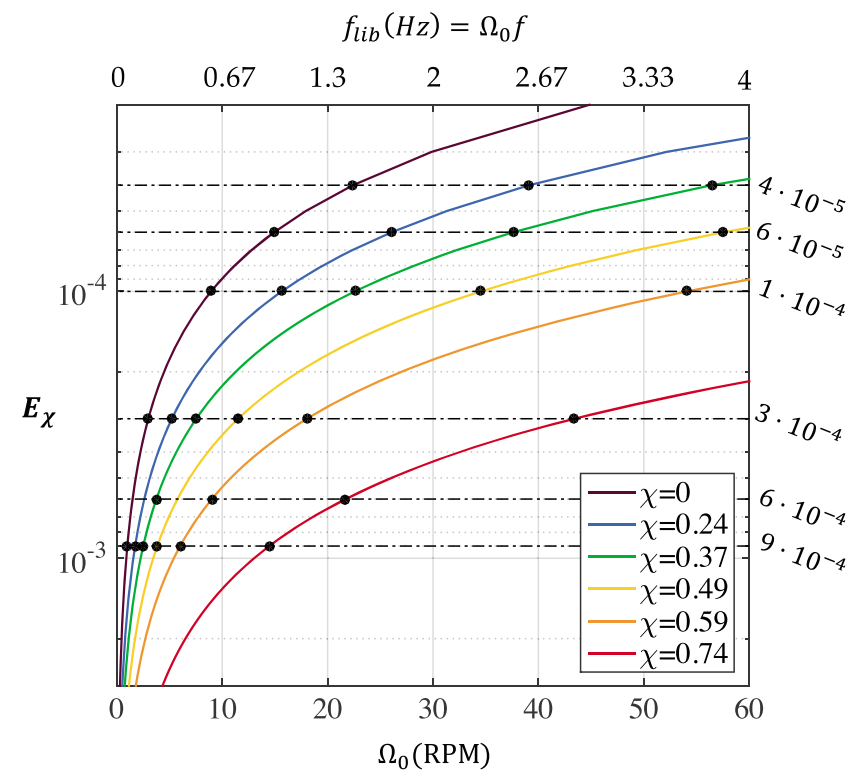

Figure 3. Evolution of the Ekman number with the rotation rate of the turntable for various shell ratios. The black dots represent the cases realized for a dimensionless libration frequency $f=4$. 
Table 1. Laboratory Experimental Librational Forcing Parameters

\begin{tabular}{lcc} 
Parameter & Definition & Range of Values \\
\hline$a$ & Long equatorial axis & $127 \mathrm{~mm}$ \\
$b$ & Short equatorial axis & $89 \mathrm{~mm}$ \\
$c$ & Rotational axis & $89 \mathrm{~mm}$ \\
$\beta$ & Ellipticity $\frac{a^{2}-b^{2}}{a^{2}+b^{2}}$ & 0.34 \\
$r_{i}$ & Inner core radius & \\
$R$ & Mean radius of the ellipsoid $\sqrt{\frac{a^{2}+b^{2}+c^{2}}{3}}$ & {$[0,25.1,38.2,50.7,61.2,76.2] \mathrm{mm}$} \\
$\chi$ & Radius ratio of the shell $r_{i} / R$ & $103.2 \mathrm{~mm}$ \\
$\Omega_{0} / 2 \pi$ & Mean fluid rotation rate & $0,0.243,0.370,0.491,0.593,0.738]$ \\
$\omega_{\text {lib }} / 2 \pi$ & Libration frequency & $0.017-1 \mathrm{~Hz}$ \\
$\Delta \phi$ & Angular displacement & $0.067-4 \mathrm{~Hz}$ \\
$v$ & Kinematic viscosity & $0.05-1 \mathrm{rad}$ \\
$f$ & Dimensionless libration frequency $\omega_{\text {lib }} / \Omega_{0}$ & $10^{-6} \mathrm{~m}^{2} / \mathrm{s}$ \\
$E_{\chi}$ & Shell Ekman number $\frac{v}{\Omega_{0} R^{2}(1-\chi)^{2}}$ & $4,2.4 \mathrm{and} f \leq 2$ \\
$E$ & Ekman number $\frac{v}{\Omega_{0} R^{2}}$ & $4 \cdot 10^{-5}-9 \cdot 10^{-4}$ \\
\hline
\end{tabular}

plane located at approximately $4.6 \mathrm{~cm}$ above the equatorial plane $(0.51 \mathrm{c})$. PIV is performed by seeding the water with $100 \mu \mathrm{m}$ diameter Optimage ${ }^{\mathrm{TM}}$ particles. Movies are acquired with the GoPro camera attached to the librating frame in a top view or side view position as shown by Figures $2 b$ and $2 c$, respectively. Note that as for Kalliroscope visualizations, the GoPro camera focuses on a given quadrant of both the vertical and horizontal planes. Frames are then extracted, converted to black and white images, and their contrast is adjusted for an optimal treatment. Computation of the instantaneous velocity fields is performed using the open source software DPIVSoft2010 [Meunier and Leweke, 2003]. The spatial resolution of the obtained velocity fields is approximately $2.5 \mathrm{~mm}$ and $2 \mathrm{~mm}$ for the horizontal and vertical planes respectively. A comparison between the results given by PIV and Kalliroscope visualizations is conducted in section 3.2.3.

\subsubsection{Analysis Methods}

To verify if the bulk turbulence appearing in our system is generated by the LDEl, we choose to perform a Fourier analysis on the direct side view visualizations. The movie analysis is performed using GoPro movies in the librating frame and MATLAB. First, a window of typically $300 \times 300$ pixels is chosen in the movie. This wide window is then typically subdivided into 36 subwindows of $50 \times 50$ pixels for which the mean intensity is calculated for each frame. This method partially removes the noise that is present when considering the signal from a single pixel. A fast Fourier transform is then performed on these 36 signals, either over a sliding average of typically 90 libration periods to see temporal changes or over larger parts of the signal to have a better frequency resolution and conduct a global analysis. We use a Hanning window to avoid spectral leakage. Finally, all these 36 spectra are stacked, once again to reduce the noise.

A similar approach is used to analyze and compare PIV with light intensity results. For each box of the PIV located inside the same window as the one defined for the Kalliroscope movies, a fast Fourier transform is performed on both the horizontal and vertical components of the velocity. The spectra corresponding to each box are then stacked.

\subsubsection{Kalliroscope-PIV Comparison}

One of the objectives of our study is to develop a method that allows for quick and easy identification of the presence of the elliptical instability. Thus, the PIV method is used to verify the results of the light intensity analysis. Figure B1 shows the results of the spectral analysis performed on both PIV and light measurements, in both vertical and horizontal planes. A direct comparison between the predominant frequency peaks shows that the analysis of the Kalliroscope visualizations can capture, qualitatively, the spectral content provided by the PIV results. Therefore, this analysis is sufficient to characterize the frequency signature of the LDEI. In the subsequent analysis, all temporal spectra are thus obtained using Kalliroscope visualizations.

\subsection{Numerical Simulations}

To complement experimental measurements, we also perform direct numerical simulations (DNSs) in the librating frame, where both the spherical inner and ellipsoidal outer boundaries satisfy a no-slip velocity 
condition. We solve the equations of motion in (4) and (5) using the spectral element solver Nek5000 developed and supported by Paul Fischer and collaborators [Fischer et al., 2007, 2008]. This method has already been used to study longitudinal libration and tides in ellipsoidal container [Favier et al., 2015; Barker, 2016; Grannan et al., 2017]. Spectral element methods have excellent convergence properties, required to simulate turbulent flows, while being able to consider complex geometries. The mesh geometry is an unstructured array of hexahedral elements, with $\mathcal{E}$ the total number of elements. Inside each element, the spectral element mesh is structured, with the variables expressed as sums of $N$ th-order Lagrange polynomials on tensor products of Gauss-Lobatto-Legendre quadrature points. In this paper, all the simulations are performed using a third-order explicit extrapolation scheme for the nonlinear convective terms and the linear inertial forces and a third-order implicit backward difference scheme for the linear diffusive term. Convergence was checked by increasing the order of the polynomial decomposition within each element.

We first perform a simulation to qualitatively confirm and compare with the experimental results. The geometry is identical to the experiment with $\beta=0.34$. We focus on a case with $\chi=0.491, f=4$, and $\epsilon=0.35$. For this relatively weak librational forcing, we are able to reach the same Ekman number as in the experiment, $E_{\chi}=10^{-4}$ (or equivalently $E=E_{\chi}(1-\chi)^{2}=2.6 \times 10^{-5}$ ). For this simulation, the mesh is composed of $\mathcal{E}=18432$ hexahedral elements with a polynomial decomposition of order $N=10$. For the spectral analysis discussed in section 4.2.1, we store the velocity components at 200 random positions within the ellipsoid, both inside and outside the tangent cylinder. The velocity is interpolated from the grid to the probe position with spectral accuracy.

Additionally, we run several simulations to study the instability close to threshold. The objective is to confirm the experimental results discussed in section 4.3. To do so, we choose the following set of parameters, $\chi=0.37$ and $f=4$, and we vary both the Ekman number and the libration amplitude $\epsilon$ in order to determine empirically the instability threshold. We start the simulations with a low-amplitude random initial condition, and we wait for the perturbations to vary exponentially with time. For these simulations, the mesh is composed of $\mathcal{E}=3840$ elements with a polynomial decomposition of $\operatorname{order} N=11$.

\section{Results}

\subsection{Basic Flow}

The theoretical basic flow (8)-(9) is compared to the experimental basic flow measured using PIV analysis. Figure 4a compares the amplitude of the theoretical basic flow with the experimentally measured basic flow, while the vector plots of the theoretical and experimental base flows are shown in Figure $4 \mathrm{~b}$. Note that in the relative error panel, the large errors located at the right of the core are due to a reflection creating a large bright patch which prevents the computation of the particle displacements. The general trend of the velocity amplitude along $s$, indicated by the black arrow in Figure $4 b$, is found to be in good agreement in Figure $4 c$. Discrepancies occur at the viscous layers close to the inner and outer rigid boundaries, which are not accounted for in the theoretical base flow. The experimental velocity amplitude is slightly lower than the theoretically predicted one because of these viscous corrections, which may also drive weak zonal flows in the bulk [Busse, 2010; Calkins et al., 2010; Sauret, 2012; Favier et al., 2015].

\subsection{Flow Visualizations and Fourier Analysis}

\subsubsection{Mode Coupling and Spatial Heterogeneity}

For all the unstable cases, the flow visualizations show a strong similarity between the full ellipsoid case and the shell case, as illustrated by the snapshots and the spectra in Figures 5 and 6 . Note that snapshots for other core sizes are provided in the appendix (Figure A1). Additionally, a video demonstration showing the early stages of the instability with and without an inner core and the corresponding numerical simulation is given in Lemasquerier et al. [2016].

We focus on the unstable cases with $f=4$ shown in Figure 5. In Figure 5a, the growth of the instability is shown in three snapshots over approximately 400 librational periods. The red and blue windows on the far right image in Figure 5 a demarcate the areas where the light intensity fluctuations are analyzed outside and inside the tangent cylinder respectively and shown in Figure $5 \mathrm{~b}$. The resulting frequency spectrograms from outside and inside the tangent cylinder are shown in Figure $5 \mathrm{c}$. Advancing through time from left to right in Figure 5, after the libration is activated, a tangent cylinder forms around the inner core and the light intensity signal is dominated by oscillations at the forcing frequency $f=4$ corresponding to the base flow. The tangent cylinder is a particular shear layer corresponding to a frequency $f=0$ for which the cone defined by the wave 
(a)

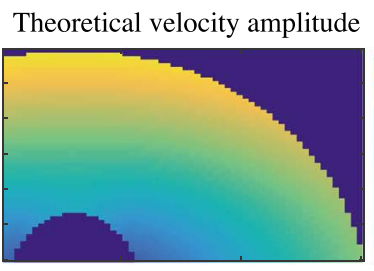

(b)
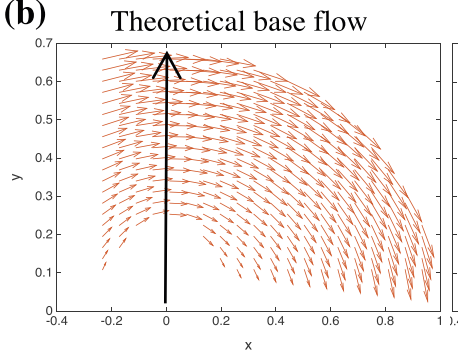

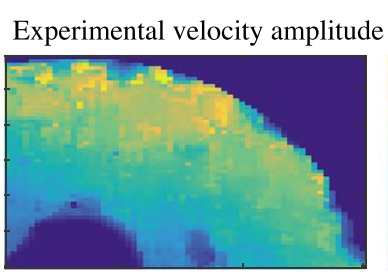

Experimental base flow

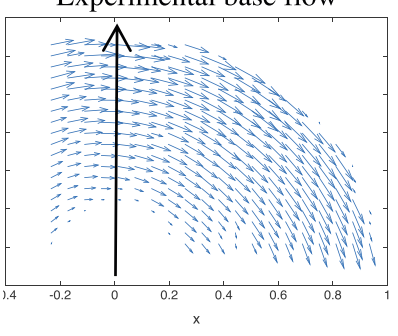

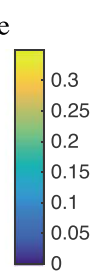
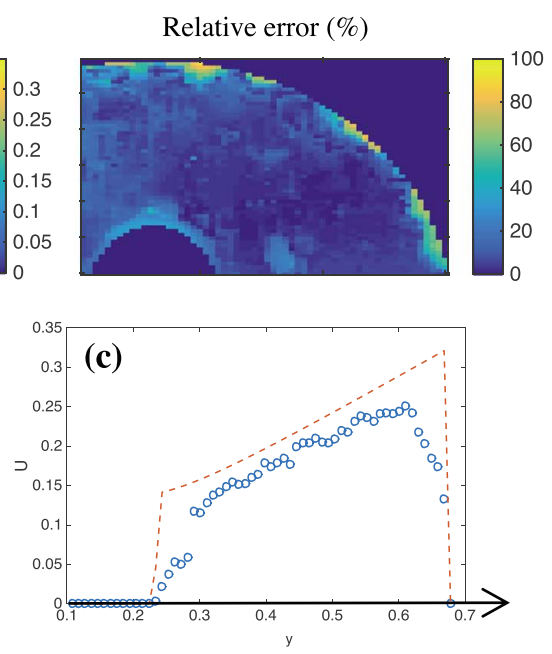

Figure 4. Comparison between the theoretical base flow in (8) and (9) and the PIV measurements. (a) Velocity amplitude. (b) Velocity field. (c) Velocity amplitude as a function of the $y$ position for $x=0$. The dashed orange line represents the theoretical velocity amplitude, and the blue circles represent the experimentally measured velocity.

packet takes the form of a cylinder $(\theta=\pi / 2)$ reminiscent of a Taylor column [Messio et al., 2008]. Then, we see the development of the pancake-like shearing structures similar to those observed by Grannan et al. [2014] and Favier et al. [2015]. Its similarity with the inertial modes found in the full ellipsoid case is confirmed by the appearance of an $\omega=2$ peak as seen in the frequency spectrograms in Figure $5 c$ between $\sim 100-150 T_{\text {lib }}$. This frequency meets the first resonance condition $\left|\omega_{1}-\omega_{2}\right|=4$. Note that on the second spectrum of Figure $5 c$, the $\omega=4$ peak seems to appear after the $\omega=2$ peak. This may be due to the lower sensibility of the Kalliroscope particles to the basic flow, as discussed in Appendix B. Finally, when the instability saturates, a wave-breaking event occurs: the resonant wave grows in amplitude until it can locally overturn or be destabilized by secondary local shear instabilities. Following this event, three-dimensional motions develop. After this breaking, the observed state of bulk turbulence is similar to the intermittent turbulence found by Grannan et al. [2014] and Favier et al. [2015] with columnar structures that are sheared by the $\omega=2$ modes as seen on the last snapshot of Figure $5 \mathrm{a}$. When the quasi steady state is reached, the $\omega=2$ peak remains, but additional frequencies $\leq 2$ appear as seen in Figure $5 \mathrm{c}$ around $200 T_{\text {lib }}$. These secondary peaks, namely, the couples [1,1], $[0.5,1.5]$, and $[0.25,1.75]$ match a resonance condition we can write as $\left|\omega_{1}-\omega_{2}\right|=2$. They could thus be the result of a secondary resonance with the primary inertial modes at $\omega \sim 2$. Such a secondary resonance has already been observed in full ellipsoids [Grannan et al., 2014; Favier et al., 2015].

This general behavior is common to every unstable case considered here. The more supercritical the instability is, the less efficient the relaminarization. However, a spatial discrimination seems to appear and becomes more obvious as the shell gets thinner. We observe that the $\omega=2$ layered structures appear above and under the inner core and extent horizontally until they reach the outer boundary. For large inner cores, the wave-breaking event always occurs primarily at the poles inside the tangent cylinder resulting in strong turbulence, whereas it does not occur as strongly in the equatorial regions outside the tangent cylinder. However, the instability still seems to grow everywhere in the bulk as seen in the second panel of Figure $5 \mathrm{a}$.

To confirm these flow differences, we performed a Fourier analysis on two different windows in the shell as represented on the last snapshot of Figure 5a. Performing a spectral analysis during the turbulent phase at these two different locations directly shows differences in terms of frequency content, as seen experimentally and numerically in Figure 6 for a shell of radius ratio $\chi=0.49$. The major difference, visible in both Kalliroscope or PIV results (Figures 6 and B1), relates to the frequencies previously identified as secondary inertial modes. Spectra computed outside of the tangent cylinder show the two couples $\left|\omega_{1,2}\right| \approx[0.25,1.75]$ and $[0.5,1.5]$, the first one being predominant. On the contrary, inside of the tangent cylinder, the couple $\sim[0.25,1.75]$ seems, if not absent, far dominated by the couple $[0.5,1.5]$. This difference is observed in all our unstable cases, except for the smallest inner core $(\chi=0.24)$.

Let us mention here that despite the peaks that we attribute to LDEl, the spectra shows other important peaks. Namely, the presence of a peak at $\omega=0.25$ is almost systematically associated with peaks at $\omega=2 \pm 0.25$ 
(a)

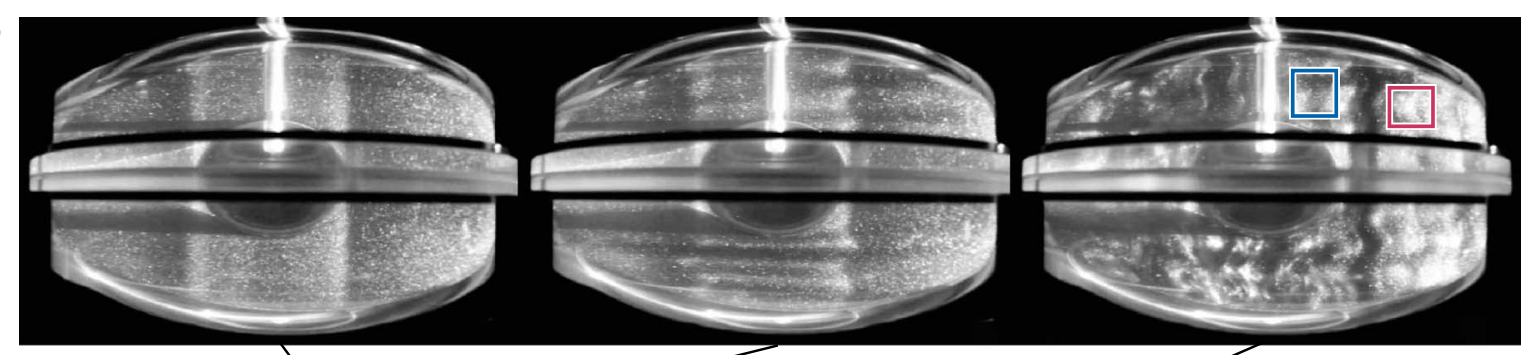

(b)
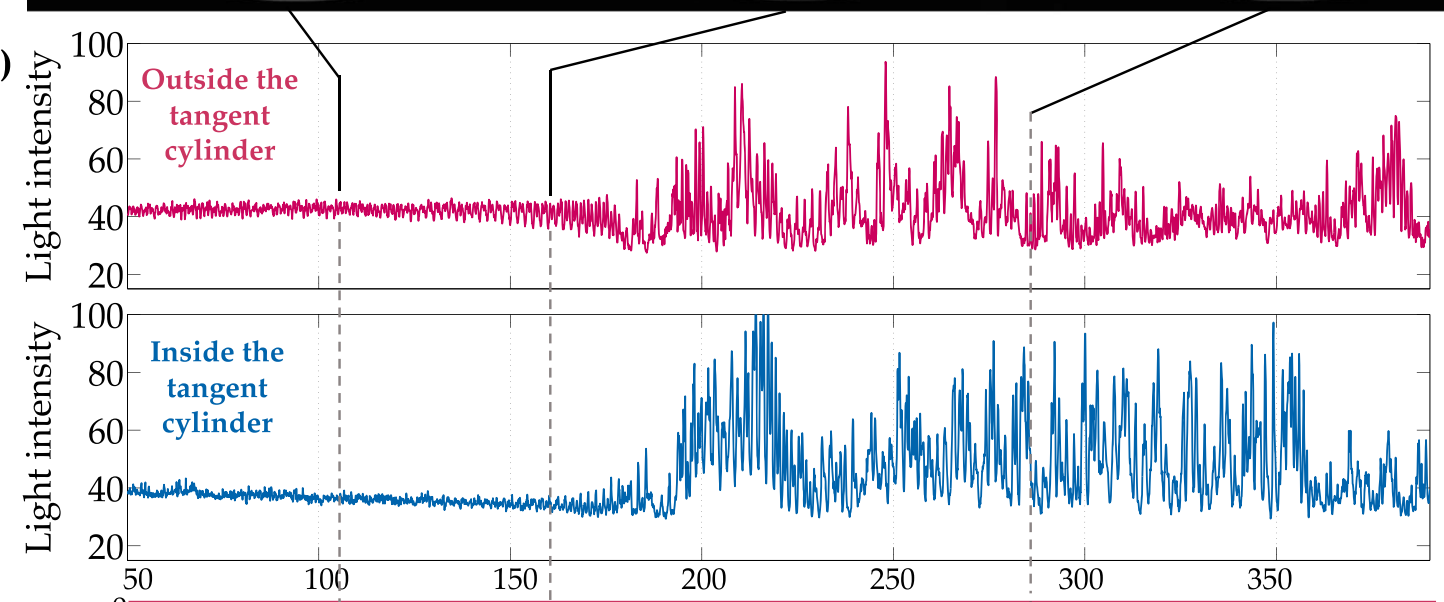

(c)

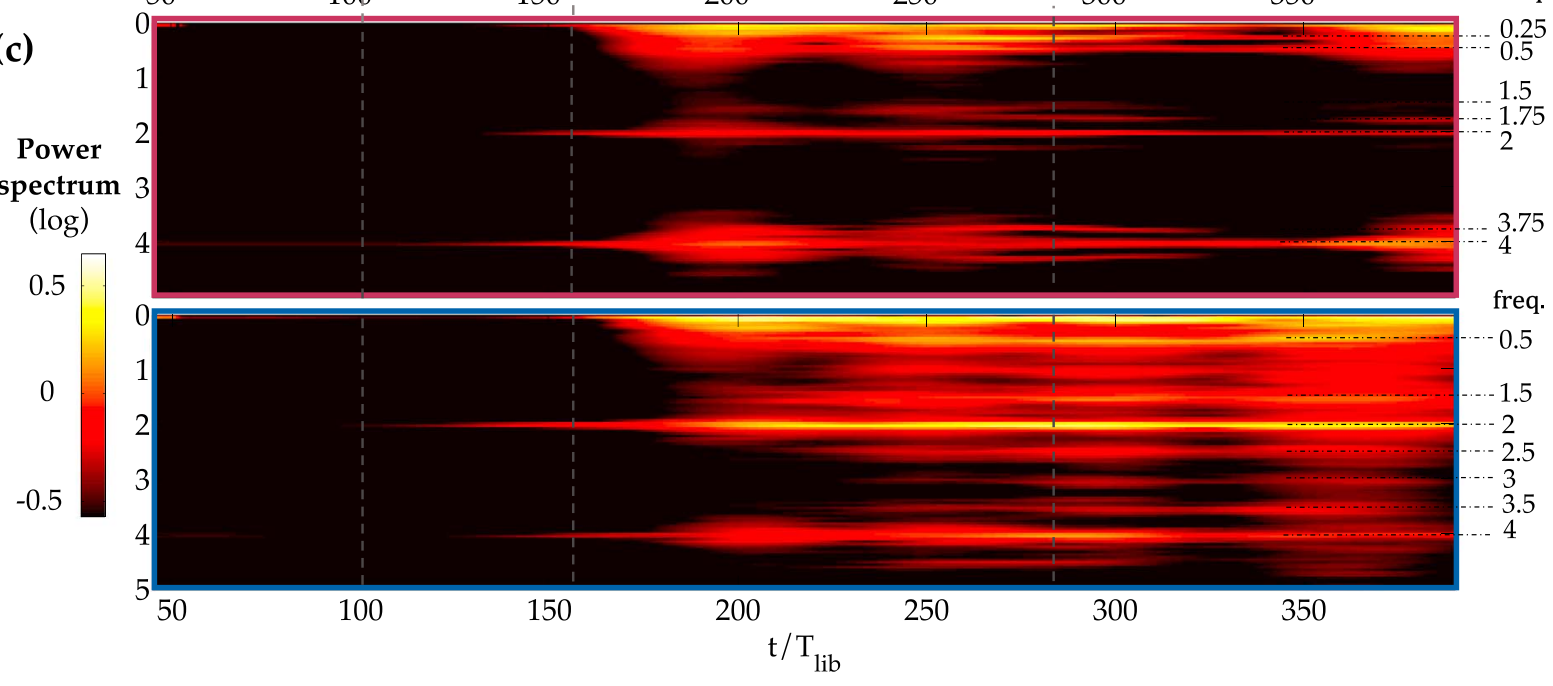

Figure 5. Light intensity analysis results for the case $\chi=0.37, E_{\chi}=6 \times 10^{-5}, \Delta \phi=5^{\circ}$, and $f=4(\epsilon=0.35)$. (a) Snapshots extracted at different times from the movie recorded by the Canon camera. (b) Light intensity signal extracted from one of the submatrices of the wide windows drawn on the last snapshot.

(c) Successive power spectra performed over a sliding window of $90 T_{\text {lib }}$ to illustrate the temporal variations of the frequency content of the signal.

and $4 \pm 0.25$, and the same coupling is observed for the peak at $\omega=0.5$. This may be due to nonlinear interactions (nonresonant) between the secondary inertial modes and the base flow or the primary inertial mode.

The same analysis is conducted in the appendix for $f=2.4$ (spin-over mode; see Appendix C). It shows the persistence of the LDEl at this particular forcing frequency.

We confirm these results with a DNS for the particular case $\chi=0.49, \epsilon=0.35$, and $E_{\chi}=10^{-4}$. First, Figure 7 shows a qualitative comparison of the onset of LDEI visualized experimentally and numerically in a meridional plane. Then, the results on inertial modes couplings are confirmed by an analysis on the numerical simulation. Velocity signals are extracted at 100 random locations during the saturated phase, both inside and outside the tangent cylinder. Figure $6 \mathrm{~b}$ shows the corresponding power spectra, averaged over all three velocity components and over all probes, where the forcing frequency at $\omega=4$ and primary resonating inertial modes 


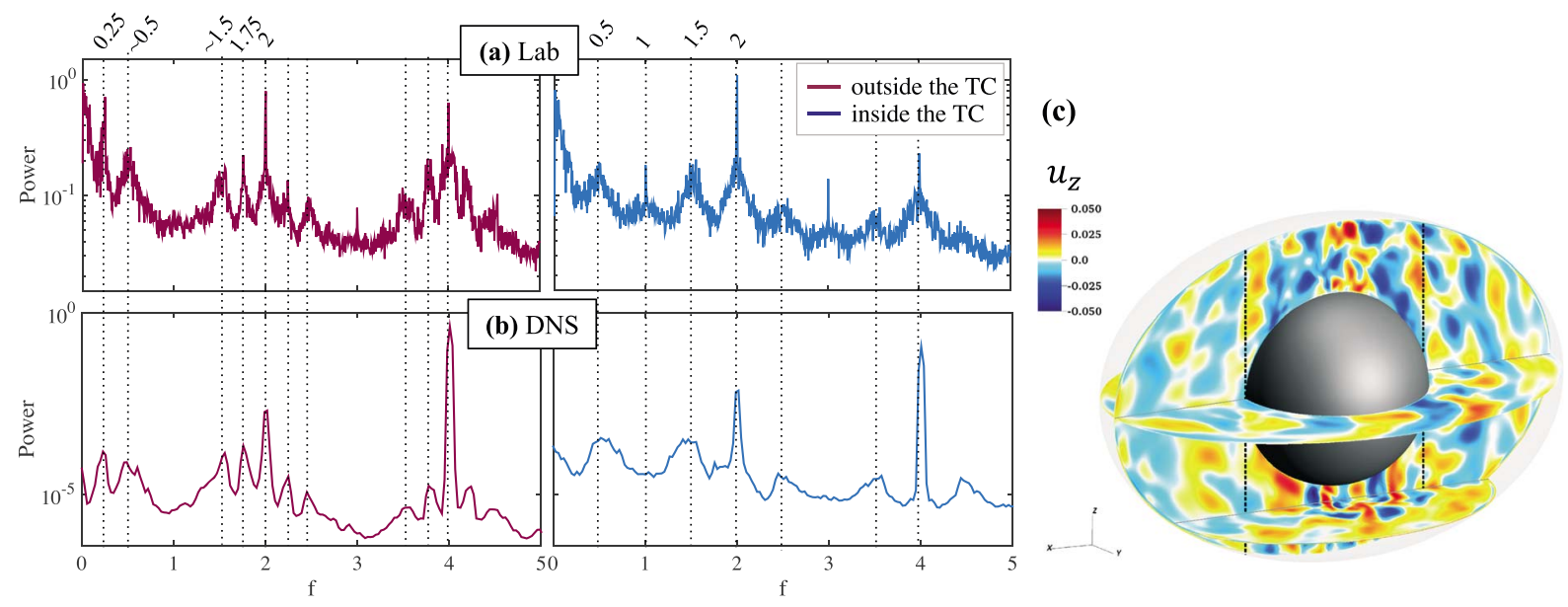

Figure 6. Analysis of the case with $E_{\chi}=10^{-4}, \chi=0.49, \Delta \phi=5^{\circ}$, and $f=4(\epsilon=0.35)$. (a) Laboratory: power spectra of the light intensity extracted from $t=200$ to $1400 T_{\text {lib }}$ on a vertical plane, both inside and outside the tangent cylinder (TC). (b) Numerics: power spectra from numerical velocity signals both inside and outside the tangent cylinder. The vertical dotted lines correspond to the frequencies of the main peaks. (c) Vertical component of the velocity during the saturated phase shown on three slices across the ellipsoidal shell (see also Figure 7 for the early stages of the instability and a comparison with the experiment). The vertical dashed lines correspond to the intersection between the tangent cylinder and the meridional plane.

at $\omega=2$ are the dominant contributions in both regions. Outside the tangent cylinder, the two dominant frequency couples are $\left|\omega_{1,2}\right| \approx[0.25,1.75]$ and $\left|\omega_{1,2}\right| \approx[0.5,1.5]$, as observed in the experimental Kalliroscope data. Inside the tangent cylinder, however, the only resonant frequencies are $\left|\omega_{1,2}\right| \approx[0.5,1.5]$. Note that the kinetic energy is typically larger inside the tangent cylinder than outside, which is confirmed by the visualization of the vertical velocity shown in Figure $6 c$. Intense overturning structures are observed above and below the inner core, whereas a relatively smooth wavefield is observed outside the tangent cylinder. To conclude, our results show that the presence of the inner core leads to significant spatial heterogeneities, in terms of both resonant frequencies and fluid motion amplitudes.

4.2.2. Influence of the Radius Ratio of the Shell $(\chi)$ and of the Ekman Number $\left(E_{\chi}\right)$

Figure 8 represents spectra realized over the turbulent phase of laboratory cases involving different inner core radii. When there is no core inside the ellipsoidal cavity, the temporal spectra are less rich and only the forcing

(a)

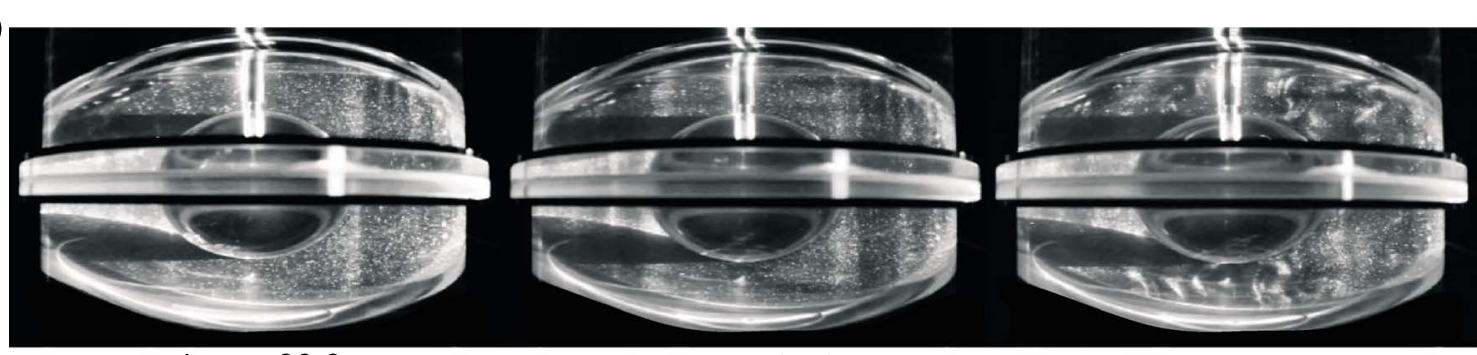

$$
t / t_{\text {spin }}=23.2
$$

$t / t_{\text {spin }}=37.2$

$t / t_{\text {spin }}=49.4$

(b)
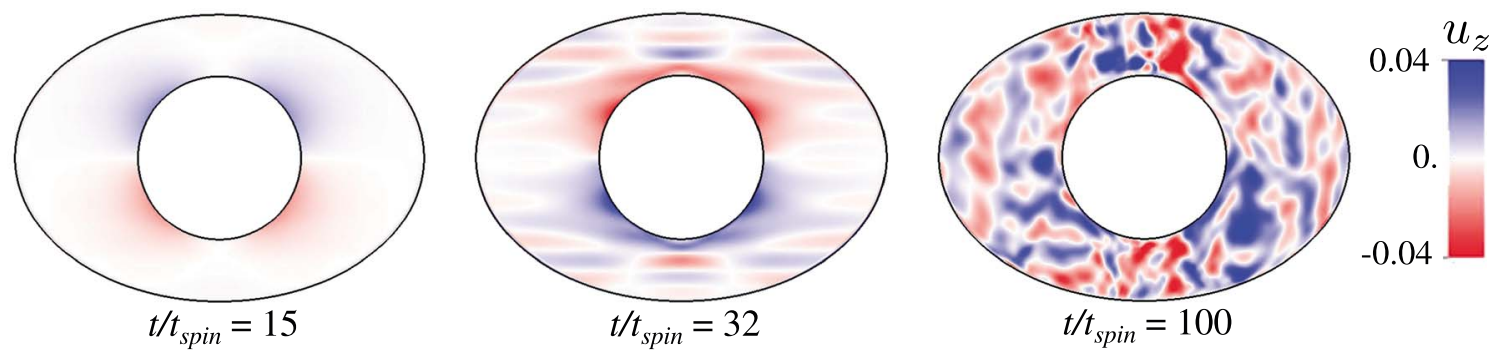

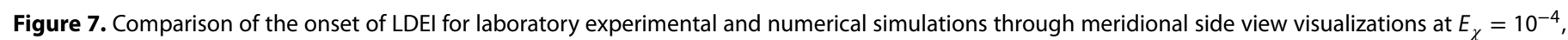

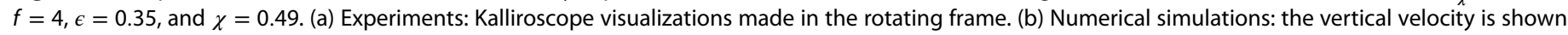
through snapshots made in the librating frame. 


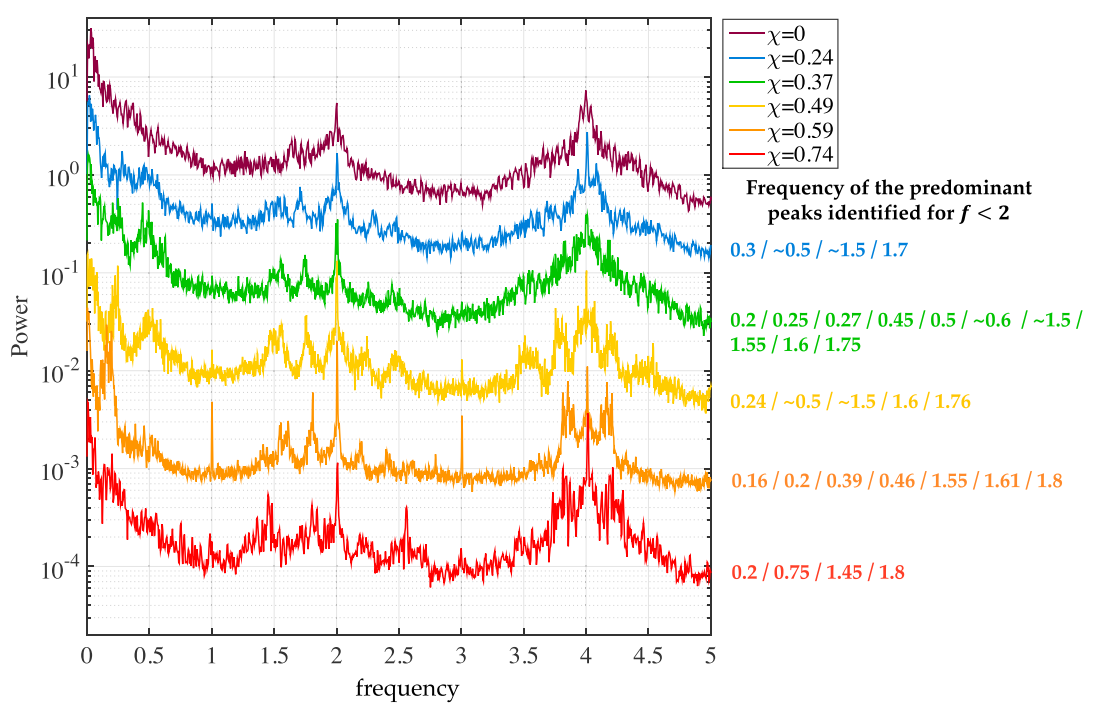

Figure 8. Power spectra of light intensity signals from outside the tangent cylinder. They are performed over the turbulent phase of cases with different inner core radius: $\left[\chi=0, E_{\chi}=4 \times 10^{-5}, \epsilon=0.28\right],\left[\chi=0.24, E_{\chi}=1 \times 10^{-4}\right.$, $\epsilon=0.35],\left[\chi=0.37, E_{\chi}=4 \times 10^{-5}, \epsilon=0.28\right],\left[\chi=0.49, E_{\chi}=1 \times 10^{-4}, \epsilon=0.35\right],\left[\chi=0.59, E_{\chi}=4 \times 10^{-5}, \epsilon=0.28\right]$, and $\left[\chi=0.74, E_{\chi}=6 \times 10^{-4}, \epsilon=0.70\right]$. The power spectra are arbitrarily shifted vertically for clarity.

frequency $f=4$ and the primary inertial modes $\left|\omega_{1,2}\right|=2$ are clearly present. The spectra are richer when a core is added, with typical frequencies around $\omega \sim 0.25,0.5,1.5$, and 1.75 as previously discussed. More interestingly, these $\omega<2$ peaks do not correspond to the exact same frequencies when comparing different $\chi$ values. For instance, the $\omega \sim 0.25$ peak is broad, spanning from $\omega_{1}=0.16$ to 0.3 , together with its companion of frequency $\omega_{2}=2-\omega_{1}$. This is reminiscent of the behavior of forced inertial modes in the spherical shell observed by Ogilvie [2009], where the dissipation at a given frequency strongly depends on the shell aspect ratio. The question remains open whether this change is due to variation in the inviscid eigenfrequency of the resonant mode or due to changes in its viscous damping.

Figure 9 compares the frequency content of two cases for which the Ekman number is significantly different. Visually, the two cases become turbulent, beginning at the poles. The $|\omega|=2$ inertial modes always remain even during the turbulent phase. The low-Ekman case, which is less viscously dominated, shows additional peaks compared to the high-Ekman case. This observation is compatible with the results of Le Reun et al. [2017], showing that an inertial wave turbulence regime-i.e., a turbulence made of the superimposition of many low-amplitude inertial waves excited by successive triadic resonances - is expected in the limit of small Ekman number.

\subsection{Instability Threshold}

Results of the libration amplitude threshold for each case are plotted in Figure 10a. Neglecting bulk dissipation, the threshold of instability is defined as the condition for which $\sigma_{\text {inv }}>K \sqrt{E_{\chi}}$. Since $f$ and $\beta$ are constant, formula (17) shows that in our case, the libration amplitude at the threshold $\Delta \phi_{\text {thres }}$ is a function of $E_{\chi}^{1 / 2}$ only (for a given $\chi$ ). Figure 10a shows that for all our cores, and even in the case of a full ellipsoid $(\chi=0$ ), this scaling is not verified. The critical libration amplitude instead varies as $\sim\left[E_{\chi}^{0.63}-E_{\chi}^{0.72}\right]$, with slight variations depending on the core considered. The numerical results represented on the same figure confirm this for the particular case $\chi=0.37$. Note that the numerical simulations predict a slightly lower critical libration amplitude compared to the experimental observations. This might be due to the presence of the shaft holding the inner sphere and interfering with large-scale inertial modes while also adding extra dissipation into the system.

Assuming a mean scaling of $E_{\chi}^{0.65}$, we deduce the corresponding dissipating factor from formula (17)

$$
\alpha \Psi(\chi)=\frac{16+f^{2}}{64} \epsilon_{\text {thres }} \beta E_{\chi}^{-0.65} .
$$

The result is represented as a function of $\chi$ in Figure 10b. The dependence with the radius ratio of the shell seems to follow the slope $\alpha(1-\chi)$ with $\alpha \sim 25$. Nevertheless, we do not claim here that this new estimate 


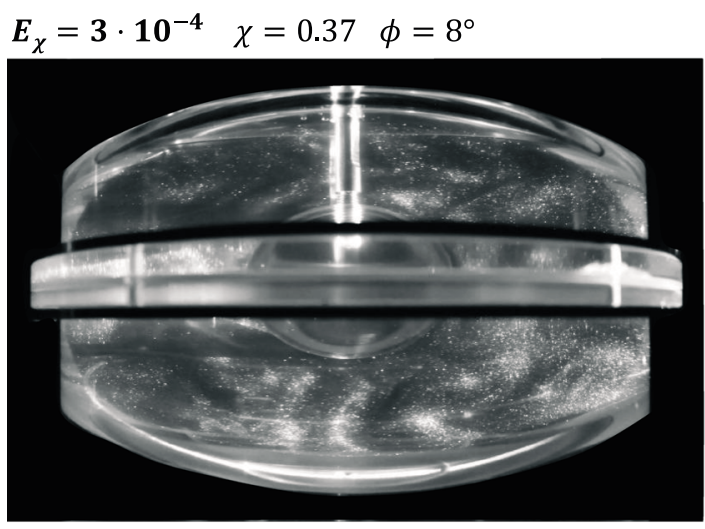

$$
E_{\chi}=6 \cdot \mathbf{1 0}^{-5} \quad \chi=0.37 \phi=5^{\circ}
$$
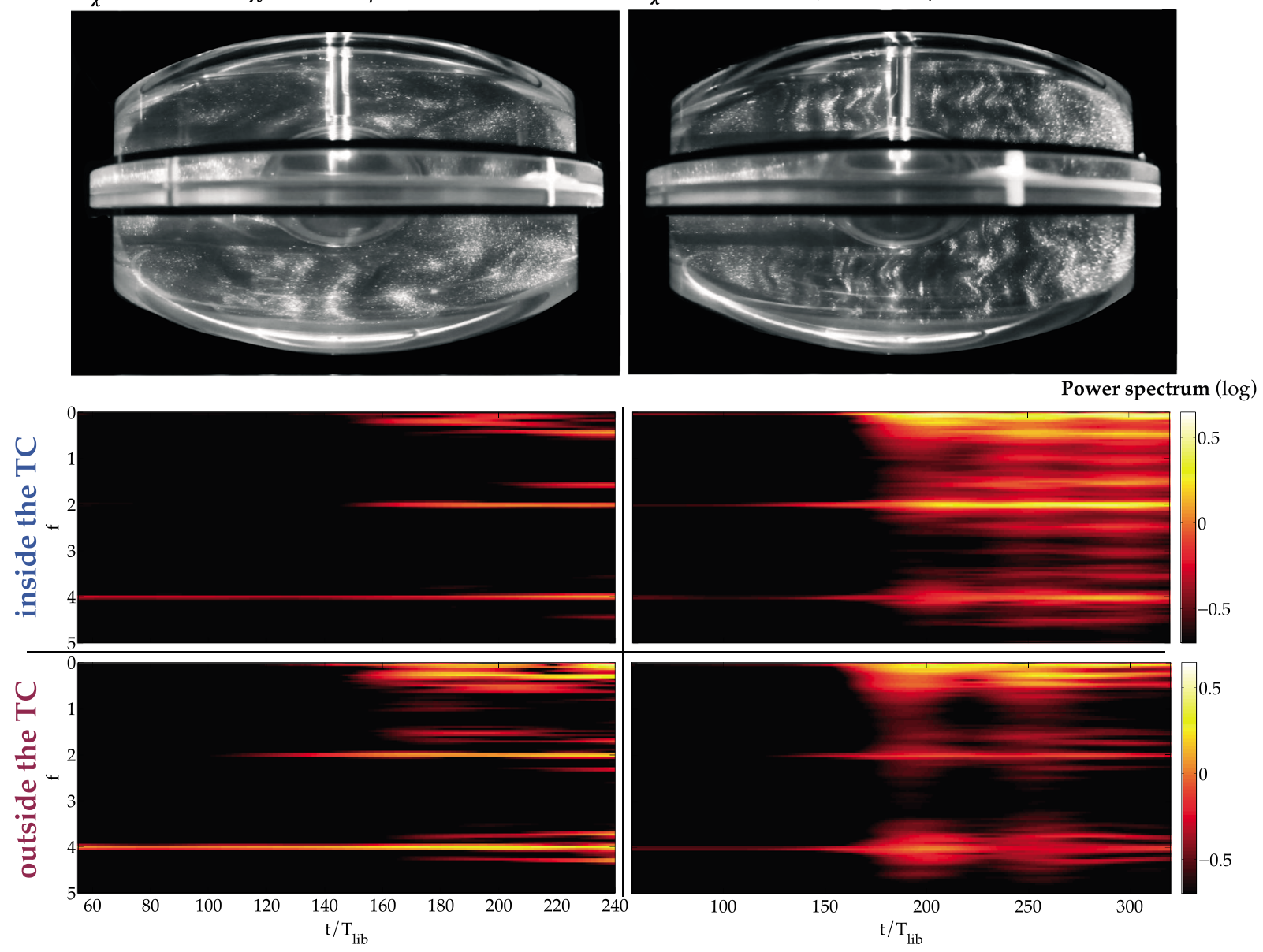

Figure 9. Successive power spectra performed over a sliding window of $90 T_{\text {lib }}$, both inside and outside the tangent cylinder (TC). The two cases correspond to the same shell and forcing parameters but at two different Ekman numbers: (right column) $\Omega_{0}=37.6 \mathrm{rpm}$ and (left column) $7.5 \mathrm{rpm}$.
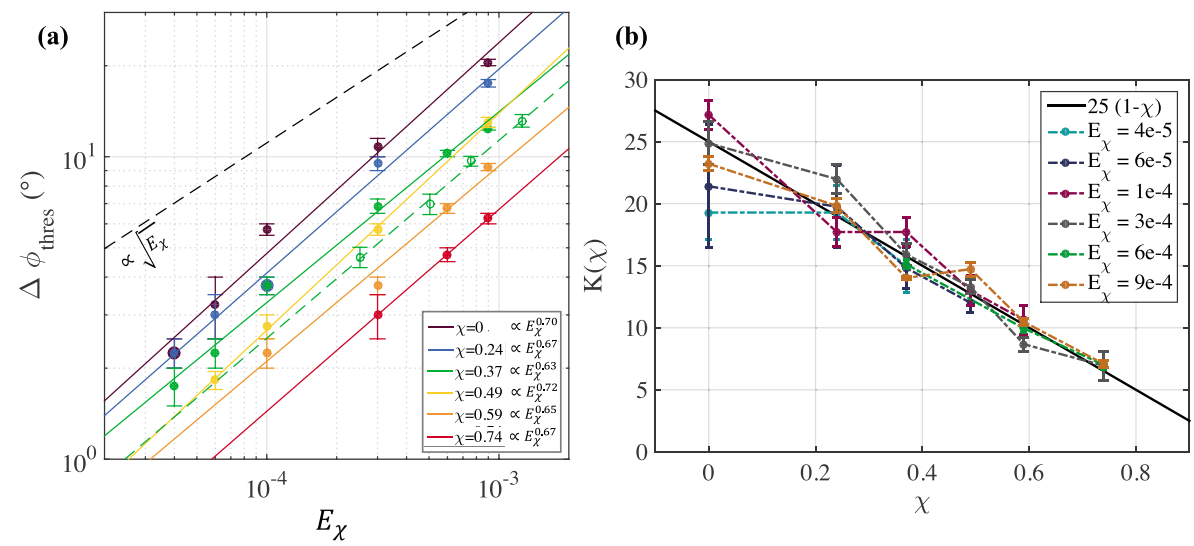

Figure 10. (a) Libration amplitudes at the threshold determined experimentally (full lines) and numerically (dashed green line). (b) Dissipation factors $K$ determined using the $\Delta \phi_{\text {thres }}$ values from Figures 10a and 18. The black line shows the main dependence with the radius ratio. 
for the threshold is universally valid: we rather think that it is valid only for the range of Ekman number explored in this study, corresponding to a transition between the regime at large Ekman number dominated by bulk dissipation (threshold scaling as $E_{\chi}^{-1}$ ) and the regime at low Ekman number dominated by boundary dissipation (threshold scaling as $E_{\chi}^{-1 / 2}$ ). This will be further discussed below in section 5.2.

\section{Discussion and Perspectives}

In this study, we first qualitatively show that longitudinal libration can directly excite inertial waves in an ellipsoidally deformed shell. Then, we used direct Kalliroscope visualizations of the flow as well as PIV to confirm the existence of the libration-driven elliptical instability (LDEI) and the related turbulent regime in a deformed shell geometry when the libration frequency is 4 and 2.4 times the rotation rate. The presence of an inner core does not strongly modify the structure of the unstable mode compared to the full ellipsoid case, at least for those two forcing frequencies. We recover the participation of inertial modes at frequencies $\pm f / 2$, and Fourier analysis suggests that we might also be able to see secondary inertial modes excited by the primary inertial modes, whose frequencies only slightly depend on the radius ratio of the shell.

However, in all our cases, the turbulence that develops in the bulk is never homogeneously distributed. Outside the tangent cylinder, a quick relaminarization occurs after the growth of the instability, but no LDEI cycle is clearly visible. Instead, the flow is dominated by geostrophic shear layers on which the inertial modes are superimposed. Besides, we notice that the growth of the instability always occurs first at the poles. These spatial heterogeneities may be due to the fact that the geometry is locally very variable in our setup. For instance, at the poles, the inner and outer boundaries are the closest, a configuration which may influence the development and resonance of inertial modes. Another interpretation is related to the heterogeneity in the effective ellipticity of the streamlines. Above and below the inner core (i.e., for $|z|>\chi$ ), the base flow is only weakly affected by the presence of the inner core so that the ellipticity is approximately uniform and equal to $\beta$. When $|z| \leq \chi$, however, the ellipticity of the streamlines is decreasing as they get closer to the inner core (see the base flow properties in section 2.2), leading to smaller growth rates (see (16) and presumably less intense flows at saturation.

The fact that the spectral content is different from the rest of the bulk suggests that the resonating inertial modes do not extend uniformly in the whole shell and may be locally stronger in the polar regions. The elliptical instability may thus induce significant spatial differences of the flow in the bulk interior, especially for a large inner core (see Figure A1).

\subsection{Inertial Modes of a Shell}

The theoretical results concerning inertial modes in a spherical shell derived by Rieutord et al. [2001] and Rieutord and Valdettaro [2010] are obtained under the assumption of a very weak forcing $(\epsilon \ll 1)$, whereas it is not the case in our experiments $(\epsilon \in[0.1,2])$. While we do observe localized shear layers generated at the inner boundary (see Figure 1), the instability discussed in this paper shares many similarities with the case of a full ellispoidal container, where resonances between regular global inertial modes are responsible for the instability [Favier et al., 2015]. In addition, the experiments and numerical simulations are currently limited to much higher values of the Ekman number than those used in theoretical studies. Thus, the relative importance between localized shear layers and global inertial modes remains to be clarified, especially when both the forcing and the Ekman number are decreased. The fact that only localized polar areas seem to resonate (see the heterogeneous nature of the resulting turbulent flow in Figure $6 c$, for example) may suggest that regular inertial modes can exist locally and that the elliptical instability can locally develop independently of the global geometry. This is reminiscent of high frequencies equatorially trapped inertial waves [Zhang, 1993] which are not affected by the presence of an inner core, although the possible link between these two problems remains to be explored. Further studies are therefore needed to assess the relevance of extending the present results to planetary conditions where the Ekman number is vanishing and the forcing is very small.

\subsection{Apparent Discrepancy Between Observed Viscous Damping and Theory}

In our experimental and numerical results shown in Figure 10a we do not recover the expected scaling law in $E^{1 / 2}$ for the Ekman numbers we consider in this study $\left(E_{\chi}=10^{-5}-10^{-3}\right)$. Instead, we predict for a libration frequency $f=4$ that the linear viscous growth rate is

$$
\sigma \approx \frac{16+f^{2}}{64} \epsilon \beta-\alpha(1-\chi) E_{\chi}^{0.65}
$$


with $\alpha \sim 25$. The origin of this scaling in $E^{0.65}$ needs to be addressed. Since it is also observed in the full ellipsoid $(\chi=0)$, the underlying mechanism is not specific to the shell geometry. Thus, it cannot be due primarily to the shear layers located at the tangent cylinder, the so-called Stewartson layers [e.g., Proudman, 1956; Stewartson, 1957, 1966; Hide and Titman, 1967; Kunnen et al., 2013]. Moreover, it is in apparent disagreement with previous studies of the viscous damping of the spin-over mode in full ellipsoids [Lacaze et al., 2004; Cébron et al., 2010a]. Focusing on the full ellipsoid case below (where the inertial modes problem is well posed), we provide a theoretical argument that the scaling in $E^{0.65}$ is possible but only for large Ekman numbers.

Since the inertial modes form a complete basis in full ellipsoids [Backus and Rieutord, 2016], we can expand the velocity perturbation $\mathbf{u}(\mathbf{r}, t)$ solution of the momentum equation (4) onto inertial modes. Using the boundary layer theory, we can determine the leading order viscous effect on each inertial mode from the inviscid solutions. Following Greenspan [1968], we expand the perturbation solution of the initial value problem as

$$
\mathbf{u}(\mathbf{r}, t)=\sum_{i} \alpha_{i}(t) \mathbf{Q}_{\mathbf{i}}(\mathbf{r}) \exp \left(\left[\mathrm{i} \omega_{i}+\tau_{i}\right] t\right)
$$

where $\left(\mathbf{Q}_{\mathbf{i}}(\mathbf{r}), \omega_{i}\right)$ are eigenvector-eigenfrequency solutions of the inertial mode problem (10)-(11), $\alpha_{i}(t)$ the modal coefficients, and $\tau_{i}$ the viscous corrections of the inviscid eigenfrequencies $\omega_{i}$. Greenspan [1968] introduces the theory up to order $E^{1 / 2}$, considering only dissipation in the Ekman boundary layer and neglecting bulk dissipation which appears at the next order $E$. One can support this truncation with the fact that inviscid inertial modes satisfy the intriguing property [Zhang et al., 2004; Vantieghem, 2014]

$$
E \int_{\mathcal{V}} \mathbf{Q}_{\mathbf{i}}^{*} \cdot \nabla^{2} \mathbf{Q}_{\mathbf{i}} \mathrm{d} \mathcal{V}=0
$$

with * indicating the complex conjugate. This volume integral is often associated with the viscous dissipation of inertial modes. However, as explained by Liao and Zhang [2008], property (21) is not physically realistic and is due to the unrealistic inviscid boundary conditions. Thus, we take into account viscous dissipation up to order $E$ to be accurate in the asymptotic expansion, extending the theory of Liao and Zhang [2008] from spheres to triaxial ellipsoids. We expand the viscous correction $\tau_{i}$ as

$$
\tau_{i}=E^{1 / 2} s_{i}+E \lambda_{i}
$$

where $s_{i}$ is the viscous correction due to the surface Ekman layer, introduced by equation (2.9.12) of Greenspan [1968], and $\lambda_{i}<0$ is the leading order volume viscous damping. The former is a complex number whose real part $\mathfrak{R}_{e}\left(s_{i}\right)<0$ is the viscous decay rate of the mode and the imaginary part $\mathfrak{\Im}_{m}\left(s_{i}\right)$ is the viscous shift in frequency of the mode. Finally, the volume damping $\lambda_{i}<0$ is proportional to the vorticity of the inviscid mode.

We have computed the first 1480 inviscid inertial modes of our ellipsoidal configuration as described by Vidal et al. [2016, 2017]. Then, in Figure 11, we show the absolute value of the viscous damping as a function of the Ekman number. Only the spin-over mode (dashed back line) and modes of absolutes frequencies $\left|\omega_{i}\right|>1.8$ (blue shading) are represented, the latter being the most excited modes for the libration frequency $f=4$. For all the modes, two limiting cases are observed: a viscous damping scaling as $E$ for large Ekman number and as $E^{1 / 2}$ for low Ekman numbers. Between these two limits there is a transition zone where surface dissipation and bulk dissipation are of the same order of magnitude. For a given inertial mode, the Ekman number of transition depends on the spatial complexity of the flow. Results for the spin-over mode shows that the damping in $E^{1 / 2}$ overcomes the damping in $E$ when $E \leq 3.10^{-2}$ (vertical dashed line). It is in agreement with previous studies [Lacaze et al., 2004; Cébron et al., 2010a], which considered the spin-over mode at Ekman numbers $E \leq 10^{-3}$. However, the scaling observed in the present study $\left(E^{0.65}\right.$, red solid line) lies in the transition zone where the two dampings play a role (depending on the excited mode). The $E^{0.65}$ scaling is due to a competition between surface dissipation and bulk dissipation. Finally, we observe in practice that the lowest Ekman number of transition depends on the number of considered modes. However, from Figure 11 we expect that the $E^{1 / 2}$ scaling may be observable for Ekman numbers $E \ll 10^{-7}$.

\subsection{Extrapolation to Planetary Interiors Conditions} 5.3.1. Libration of Planetary Bodies

When thinking of planetary applications, the first question to arise is the validity of our experimental setup to model the libration forcing of different bodies. Namely, the shape and the movement of the inner core have to be discussed. For a given body, if both the outer and the inner boundaries are ellipsoidal (e.g., the mantle and the solid inner core of Mercury), they should both undergo the same libration forcing. Apart from 


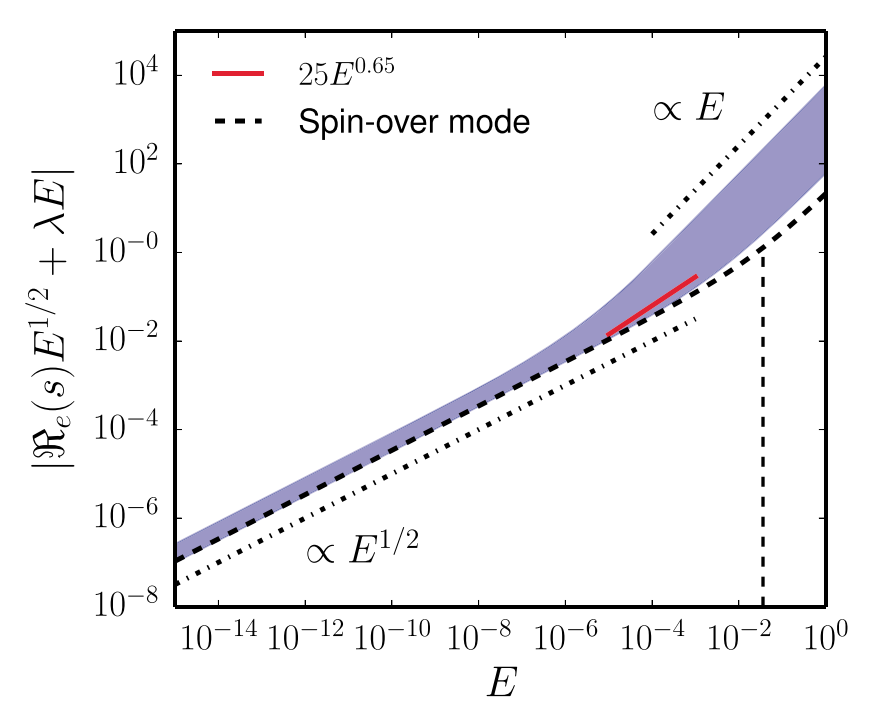

Figure 11. Viscous damping of inertial modes as a function of the Ekman number $E$ for the first 1480 inertial modes of our full ellipsoid. The viscous damping is a combination of the surface Ekman layer damping $\Re_{e}(s) E^{1 / 2}$ and the bulk viscous damping $\lambda E$. We only show the spin-over mode (dashed back thick line) and the first 140 modes of frequencies $\left|\omega_{i}\right|>1.8$ (blue shading), which are the most excited modes for $f=4$. The vertical dashed line shows the Ekman number for which volume and surface dampings of the spin-over mode are equal. Slopes of asymptotic behaviors associated with surface and volume dampings are also shown. The surface damping only dominates when $E \ll 10^{-7}$.

the forcing, the movement of these two boundaries is also constrained by their coupling, which might be of a viscous, electromagnetic, or gravitational nature [e.g., Yseboodt et al., 2013]. However, both the amplitude of the inner boundary deformation and the amplitude of a supposed coupling are unknown. Due to these uncertainties, we chose the simplest possible experimental setup: a spherical inner core that librates with the same amplitude and frequency as the outer boundary. Technically, fixing the rod that suspends the core to the librating container is indeed easier. Besides, in our setup, the core is spherical; thus, the coupling between the inner core and the fluid is purely viscous. The difference between a librating and a static spherical inner core or a spherical inner core librating at a different rate should thus be negligible in the limit of low planetary Ekman numbers (see, e.g., Calkins et al. [2010] for a study with spherical boundaries). Choosing an ellipsoidal inner core may modify the inhomogeneities described in section 4.2.1 and may also drive supplementary instabilities. Thus, the system could have even richer dynamics. In that sense, our choice of a spherical inner core suggests that our present study provides a simplified lower bound for elliptical instabilities that can arise in a librating planetary fluid shell.

\subsubsection{Stability Analysis}

Apart from the question of the existence and the form of inertial modes in a given geometry, local stability analysis can be used to evaluate the presence of the elliptical instability in terrestrial bodies. In terms of stability analysis, in the regimes experimentally and numerically explored in this study the usual scaling $\sqrt{E}$ is not verified because of bulk dissipation. However, it holds when extrapolating to planetary conditions. Thus, for $f=4$, and in the range of parameters of this study, the growth rate $\sigma$ is

$$
\sigma \approx \frac{16+f^{2}}{64} \epsilon \beta-\alpha(1-\chi) E_{\chi}^{0.65}
$$

with $\alpha \sim 25$, whereas for $E \ll 10^{-7}$,

$$
\sigma \approx \frac{16+f^{2}}{64} \epsilon \beta-\alpha \sqrt{E}
$$

Extrapolating from the experimentally determined $E^{0.65}$ slope, we choose $\alpha \sim 3$ for planetary applications, i.e., for $E \ll 10^{-7}$ (see Figure 12). The criterion of instability $\sigma>0$ is plotted as a function of $E$ in Figure 12.

Knowing the parameters involved in these equations for a given interior layer of a body thus allows the estimation of whether it is theoretically unstable or not (criterion $\sigma>0$ ). We apply this criterion to the four Galilean moons (Io, Europa, Ganymede, and Callisto), two moons of Saturn (Titan and Enceladus), and three Super-Earths expected to be telluric ( $55 \mathrm{CnC}$ e, CoRoT-7b, and GJ 1214b). All the bodies considered here 


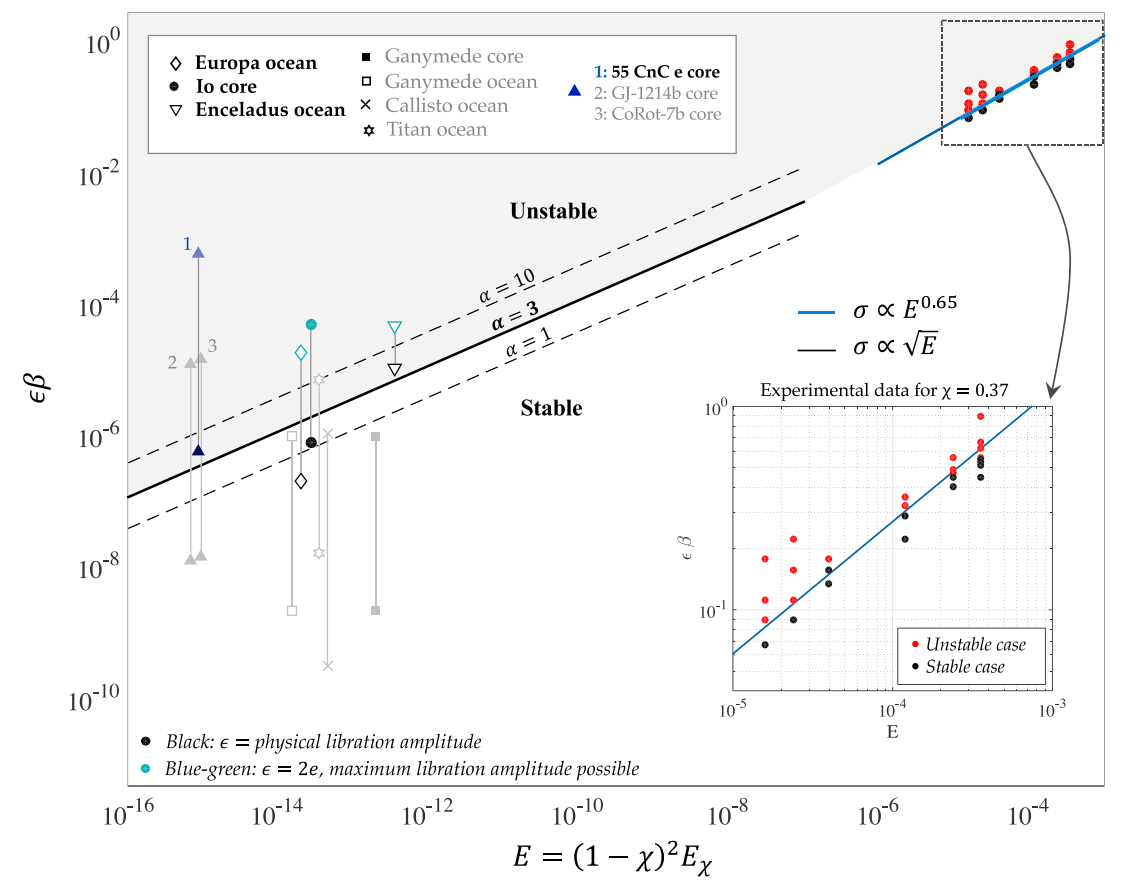

Figure 12. LDEI stability diagram. For each body, the vertical line represents the range from a libration amplitude equals to the physical libration up to the optimal libration amplitude $2 e$. The bodies for which LDEI is likely absent are plotted in light gray. The oblique lines represent the criterion $\sigma>0$, using equation (23) for $E \gg 10^{-7}$ and equation (24) for $E \ll 10^{-7}$. The $E^{0.5}$ scaling is justified by the hypothesis that the dissipation occurs mainly in the Ekman boundary layer. In our experimental survey (upright corner), the $E^{0.65}$ scaling is due to a transition toward a regime for which bulk dissipation becomes more important. Note that for the regimes explored experimentally, the value of $\epsilon \beta$ at the threshold depends on $\chi$ (see equation (23). That is why it is only represented for $\chi=0.37$. The dashed lines represent two extreme values for $\epsilon \beta$ at the threshold, using $\alpha=[1,10]$. The gray space is the unstable region.

are in synchronous rotation, their mean rotation period being equal to their orbital period (librations of dimensionless frequency $f=1$ ).

The maximum amplitude of libration is theoretically equal to the amplitude of the variations of the orbital velocity, i.e., $2 e$, where $e$ is the orbital eccentricity. However, this is an optimal case which implies that the spin rate of the body is so slow or the body is so elastic that it has the time to completely adapt to the gravitational constraints. This maximal libration is called the optical libration. However, because of the rigidity of the outer boundary of the shell and of the spin rate, the amplitude of the differential rotation $\epsilon$ between the fluid and the librating static bulge is smaller than $2 e$.

Finally, because the equatorial ellipticity $\beta$ of the considered fluid layer is generally unknown, Cébron et al. [2012b] estimate it by assuming a hydrostatic equilibrium shape, which gives

$$
\beta=\frac{3}{2}\left(1+k_{2}\right) \frac{M}{m} \frac{R^{3}}{D^{3}}
$$

where $m$ and $R$ are respectively the mass and the mean radius of the considered body with a potential Love number $k_{2}$ and $D$ the distance between the body and its attractor of mass $M$. This method is used for every planetary body considered in this study, except Enceladus. Table D1 gives the values used to calculate $\epsilon \beta$ for each body. Figure 12 represents the position of these bodies compared to the theoretical threshold extrapolated from our results when assuming an $E^{0.5}$ scaling for $E \ll 10^{-7}$.

This scaling shows that Enceladus' ocean is expected to be unstable with a good level of confidence, even when considering the uncertainty about its equatorial ellipticity (see Table D1). Besides, since $\chi=0.84$ (calculated from Beuthe et al. [2016]), we expect strong spatial heterogeneities for the LDEI between the poles and the equator, as seen when varying the size of the inner cores used in our experiments. Such latitudinal variations in turbulent mixing may prove to be relevant to transport phenomena on such bodies. For Enceladus' ocean, the ocean dynamics may influence the ice-ocean interface dynamics, namely, by generating nonhomogeneous heat fluxes in the fluid layer [see, e.g., Aurnou et al., 2008]. This might help to explain 
the variations of Enceladus' ice shell thickness, which is modeled by Beuthe et al. [2016] to be $23 \pm 4 \mathrm{~km}$ thick in average, but reduced at the poles (up to $7 \pm 4 \mathrm{~km}$ at the South Pole). However, further quantification of spatial heterogeneity of librationally driven turbulence in relatively thin ellipsoidal shells is still needed in order to make detailed planetary predictions.

Then, an elliptical instability is possible but uncertain for other fluid layers like lo's core and Europa's ocean since they are near the threshold when considering their physical libration amplitude. However, the libration amplitude of the icy shell of Europa is taken from Van Hoolst et al. [2008] but has not been measured yet with accuracy. That is why for Europa's ocean, the whole range of values has to be considered, and it has a nonnegligible chance to be unstable. It is improbable that the core of Ganymede and the subsurface oceans of Callisto and Ganymede are unstable, considering their proximity with the threshold. The same uncertainty is observed for the subsurface ocean of Titan, which is nevertheless more likely unstable. Finally, the (supposed) liquid cores of the three exoplanets considered are likely unstable because of their close orbit around their stars. The physical libration amplitude is here arbitrary taken as 3 orders of magnitude lower than the optical libration.

\subsection{Perspectives and Open Questions}

Our study focuses on the particular case for which the librating forcing $(f=4)$ indirectly excites inertial waves propagating quasi-horizontally. However, a look at 12 different forcing frequencies shows that this also excites $f \leq 2$ inertial waves, observed via the formation of oblique shear layers in the flow (Figure 1). Besides, we observe that an instability develops at the poles where the characteristics converge and that differential rotations are generated in the bulk (geostrophic shear layers). It may thus be of interest to conduct quantitative studies in this regime to estimate, for instance, dissipation rate, to verify the width of the shear layers and its scaling with Ekman, to measure the amplitude of the flow and to qualify the associated nonlinearities such as the generation of zonal flows [Favier et al., 2014].

It is now of primary importance to determine whether the elliptical instability persists for other forcing frequencies. Theoretically, all forcing frequencies between 0 and 4 should give rise to LDEl, in the limit of small Ekman. For now, we have simply verified that it was the case for $f=4$ and $f=2.4$ (Figures 5 and C 1 ), and our analysis suggests the same conclusion for $f=1.6$ for which we identify at least a coupling between inertial modes of frequencies $\omega_{1,2}=[0.35,1.25]$ (not shown). Quantitative studies are also needed for a fine characterization of the nonlinear turbulence following the growth of the instability and to verify and interpret the flow spatial differences observed in the bulk [e.g., Le Reun et al., 2017].

Finally, we show that the elliptical instability occurs in ellipsoidal shells. The associated instability criterion has been described in this study in the case of longitudinal libration. Further studies are needed to define the instability criteria of latitudinal libration (e.g., Vantieghem et al. [2015] in the case of a full ellipsoid) and tidally driven elliptical instability in ellipsoidal shells [Lacaze et al., 2005; Grannan et al., 2017], which may be less restrictive. Moreover, it has been recently observed that the orbital eccentricity favors elliptical instabilities [Vidal et al., 2017].

More generally, if they exist, the importance of these mechanically driven turbulent motions needs to be addressed. They may be of geophysical relevance for the following:

1. Energy dissipation: The dissipation induced by direct and indirect tidal or librational resonances of fluid layers may play a role in the rotational or orbital dynamics of the considered planetary system [Le Bars et al., 2015]. The relative importance of direct forcing compared to the elliptical instability also needs to be investigated.

2. Ocean stratification: Turbulent mixing may indeed lead us to question the stratification of subsurface oceans and the possibility for hosting life there.

3. Core stratification: It has been proposed that the supposed stratified layer at the top of Earth's core is the result of the Moon-forming impact [Landeau et al., 2016]. However, after impacts, the strong perturbations of rotation may be able to mechanically mix out chemical stratifications. The relative importance of such a mechanical mixing compared to a possible convective mixing [see, e.g., Levy and Fernando, 2002] also needs to be determined.

4. Dynamo action: This type of instability may provide an important piece that explains how dynamos are sustained when the thermosolutal convection models are insufficient. See, for instance, Wu and Roberts [2013] for dynamo driven by longitudinal libration, Le Bars et al. [2011] and Dwyer et al. [2011] for the past dynamo of the Moon, and Arkani-Hamed et al. [2008] for Mars. 

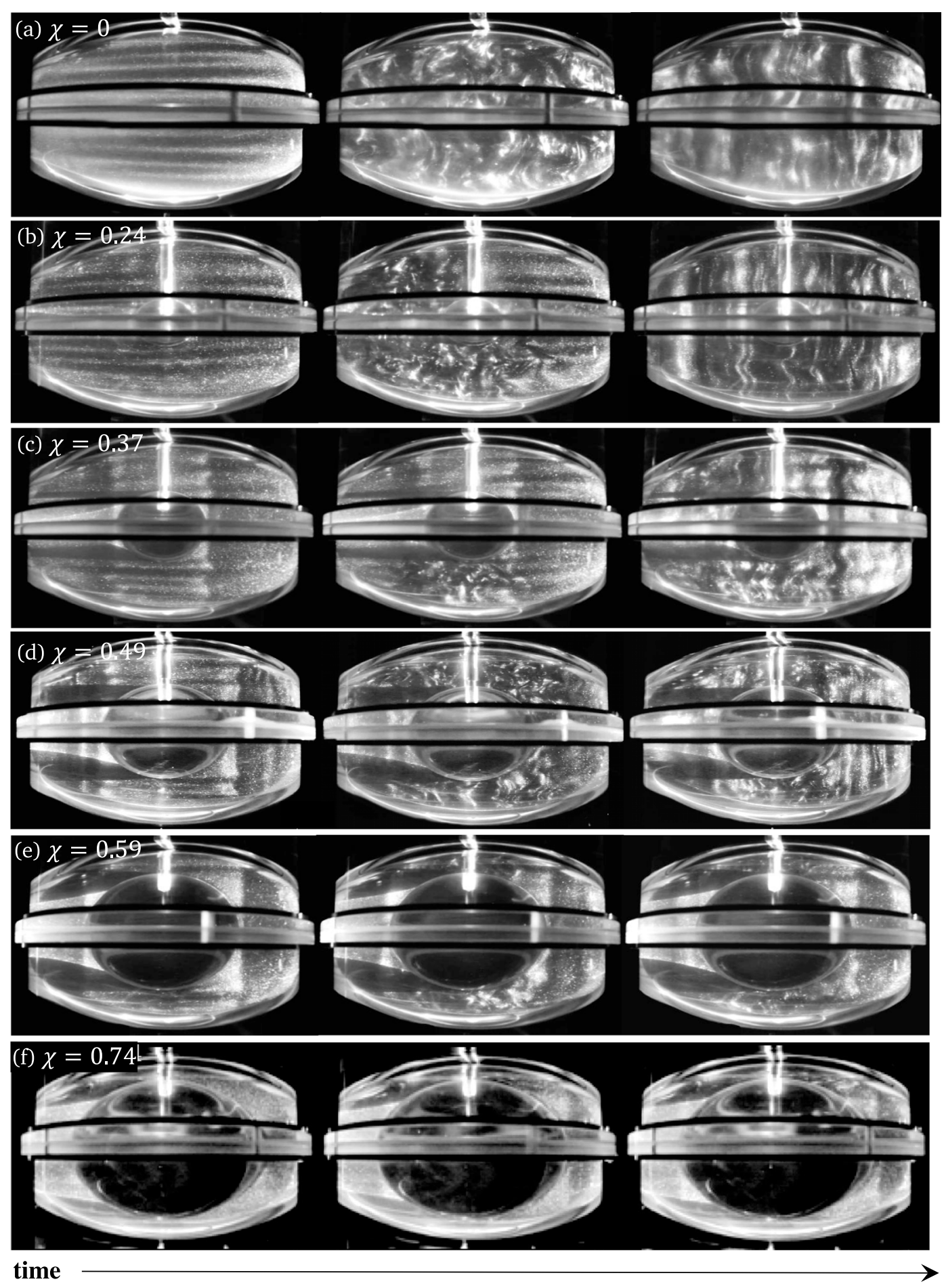

Figure A1. Typical flows observed in unstable cases. For each $\chi$, the three snapshots are in the chronological order. The first snapshot shows the development of the $f_{1,2}=2$ inertial modes. The second snapshot shows moments where strong turbulence is observed. This state can be very short since the relaminarization occurs quickly, that is why the turbulence is shown at moments when it is not yet uniform in the whole shell. The last snapshot illustrates the saturation state, with dominant columnar sheared flow outside the tangent cylinder and turbulence inside. These frames are extracted from the Canon EOS 7D movies. (a) $E_{\chi}=6 \times 10^{-5}, \Delta \phi=10^{\circ}$; (b) $E_{\chi}=6 \times 10^{-5}, \Delta \phi=5^{\circ}$; (c) $E_{\chi}=6 \times 10^{-5}, \Delta \phi=5^{\circ}$; (d) $E_{\chi}=1 \times 10^{-4}, \Delta \phi=7.5^{\circ}$; (e) $E_{\chi}=3 \times 10^{-4}, \Delta \phi=12.5^{\circ}$; and (f) $E_{\chi}=9 \times 10^{-4}, \Delta \phi=10^{\circ}$. 


\section{Appendix A: Additional Side View Visualizations for $f=\mathbf{4}$}

In Figure A1, snapshots for six different experimental cases are provided. Each line corresponds to a case with a different inner core size, beginning with no inner core. This figure illustrates visually the spatial heterogeneities of the flow generated by the presence of an inner core and its reinforcement as the inner core gets larger. A video demonstration showing the early stages of the instability with and without an inner core is given in Lemasquerier et al. [2016].

\section{Appendix B: Validation of Kalliroscope Results by PIV Analysis}

PIV method is used here to verify the information content of light intensity analysis. Figure B1 shows the result of the spectral analysis performed on PIV and light measurements, both in vertical and horizontal planes. The fast Fourier transform was applied on the signal from the moment when the flow becomes turbulent until the end of the acquisition. The interpretation of the observed peaks is conducted in section 4.2.1.

The main difference concerns the relative peak sizes, the PIV data being far dominated by the base flow, whereas it is not the case for the light intensity signal. This is not surprising since the base flow is a coherent flow which does not generate any strong velocity gradient nor shearing zone. Thus, it does not create important light contrast in a flow seeded by Kalliroscope particles. Also, the base flow is at rather high frequency and is intrinsically less obvious from Kalliroscope particles that need time to align with a given shear. The only source of this signal is thus due to the periodic reorientation of the Kalliroscope particles which generates slight light intensity variations. On the contrary, in terms of velocity amplitude, the base flow is very strong and predominates the velocity signal, particularly on a horizontal plane. We conclude from this that one has to be very careful on the relative peak intensity seen in spectra extracted from a light intensity analysis because it depends on the geometry of the flow considered. The Kalliroscope data also show a strong component at zero frequency, due to the ambient light intensity even in the absence of motion (see, e.g., Figure 5). That being said, Figure B1 shows that the relative amplitudes of the peaks are qualitatively similar for both methods.

The last main difference is that light intensity signals show an artifactual $\omega=1$ frequency (and its harmonic at $\omega=3$ ), which corresponds to the rotation rate $\Omega_{0}$. This is probably the consequence of light variations due to
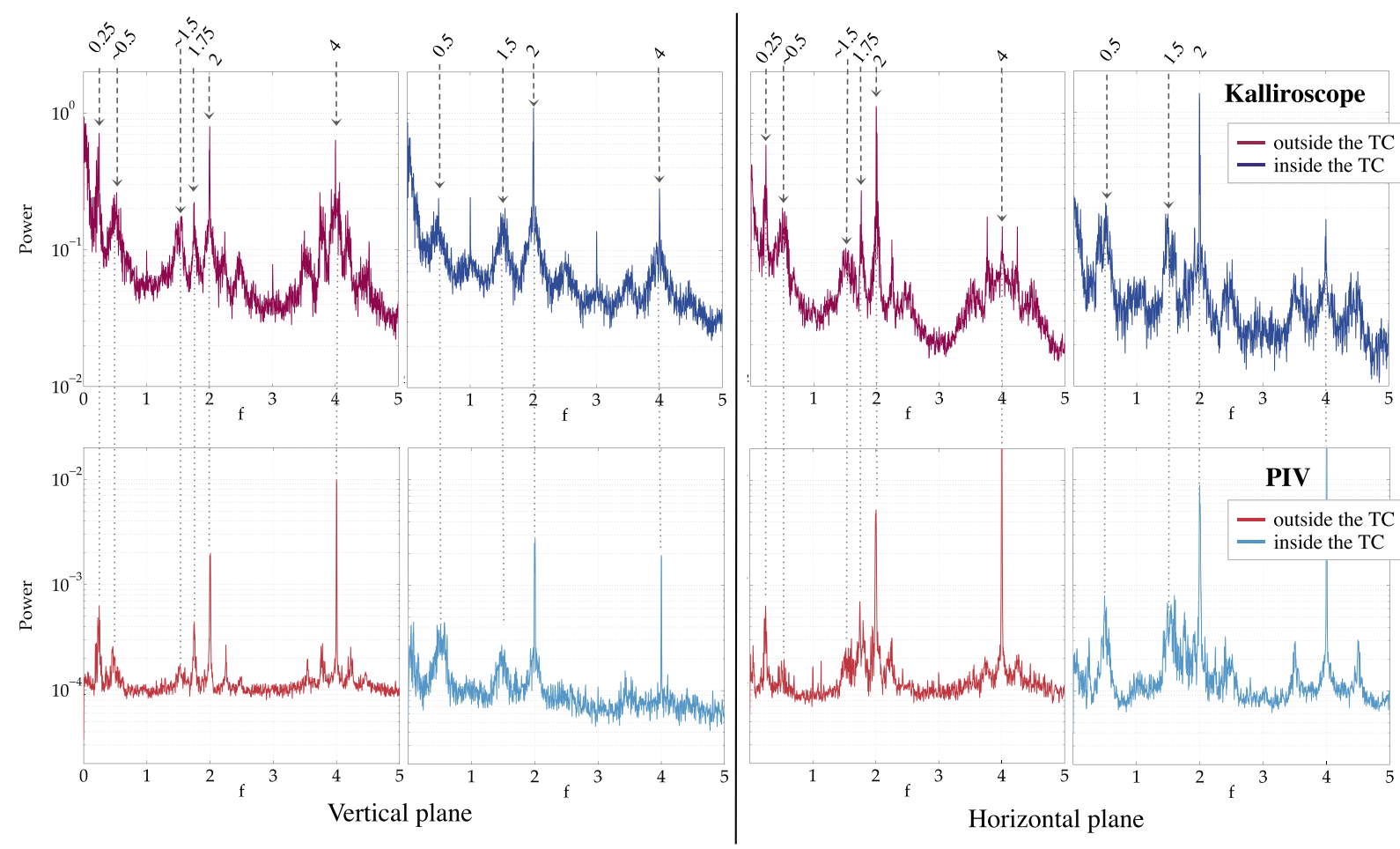

Figure B1. Power spectra of the light intensity and velocity signals from $t=200$ to $1400 T_{\text {lib }}$, both inside and outside the tangent cylinder (TC). The case parameters are $E_{\chi}=1 \times 10^{-4}, \chi=0.49, \Delta \phi=5^{\circ}$, and $f=4(\epsilon=0.35)$. (top row) Light intensity results and (bottom row) PIV results. 
the nonuniformity of the environment surrounding the experimental setup or to an external source of light in the experimental room.

\section{Appendix C: Excitation of the Spin-Over Mode $(f=2.4)$}

No survey has been realized for a libration forcing $f=2.4$. However, we show here the persistence of the LDEI at this forcing frequency. Figure $\mathrm{C} 1$ shows the light intensity analysis results for a typical unstable case. When the periodic forcing is activated, the tangent cylinder appears as well as inertial shear layer, as can be seen on the first snapshot of Figure $\mathrm{C} 1 \mathrm{a}$. Figure $\mathrm{C} 1 \mathrm{c}$ shows that in terms of frequency, inertial modes of frequency $\omega / 2= \pm 1.2$ are indirectly excited. Such a frequency would give shear layers emitted from a critical latitude $\theta_{c} \approx 37^{\circ}$ with an angle $\alpha_{H} \approx 53^{\circ}$ from the horizontal, which is in good agreement with the geometry of the observed shear layers.

Visually, the first instability develops at the two poles. This instability spreads slowly, and the sides also becomes unstable (second snapshot of Figure C1a) before the classical " $\mathrm{S}$ " shape of the spin-over mode

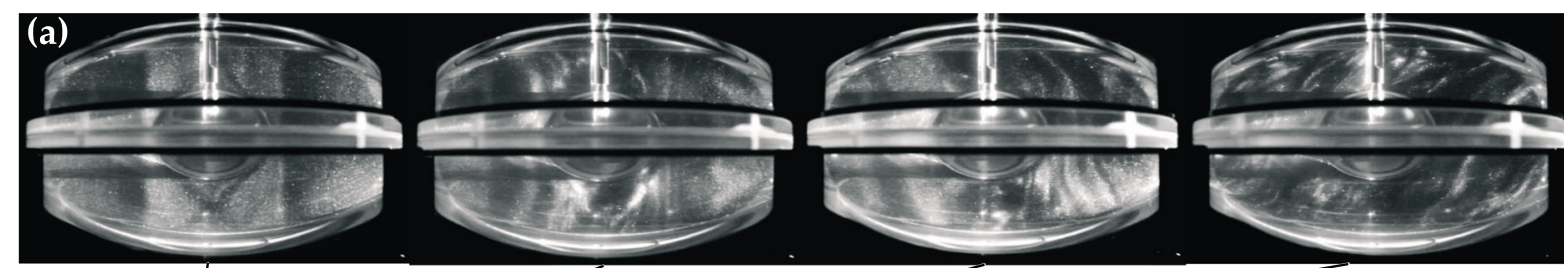

(b)

b)
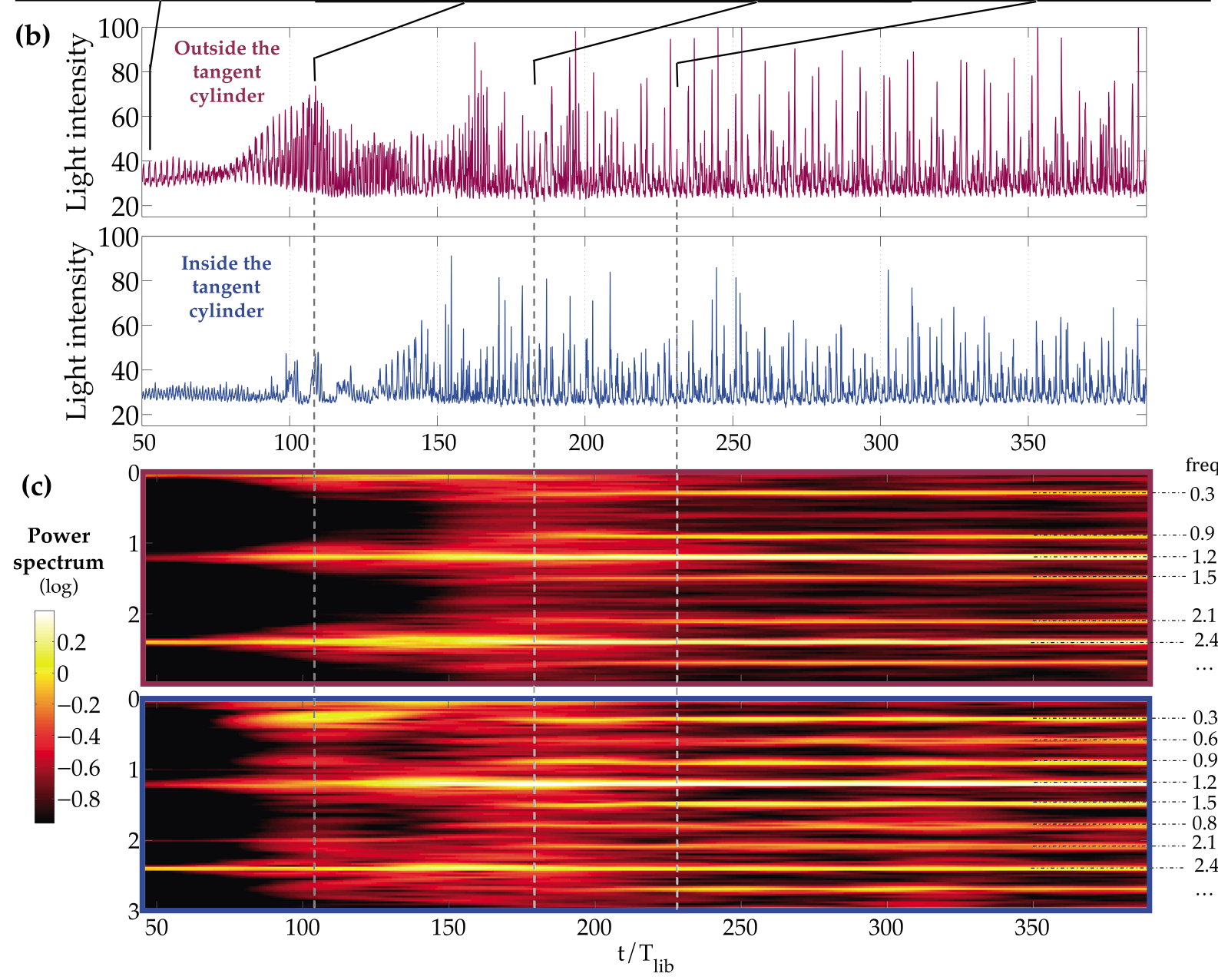

Figure C1. Light intensity analysis results for the cases $\chi=0.37, E_{\chi}=1 \times 10^{-4}, \Delta \phi=15^{\circ}$, and $f=2.4(\epsilon=0.63)$. (a) Snapshots extracted at different times from the movie recorded by the Canon camera. (b) Light intensity signal extracted from one of the submatrices. (c) Successive power spectra performed over a sliding window of $90 T_{\text {lib }}$ to illustrate the temporal variations of the frequency content of the signal. 
Table D1. Physical Characteristics Used for the Stability Analysis ${ }^{a}$

\begin{tabular}{|c|c|c|c|c|c|c|c|c|c|}
\hline & $10^{b}$ & Europa $^{b}$ & Ganymede $^{b}$ & Callisto $^{b}$ & $\operatorname{Titan}^{\mathrm{b}}$ & Enceladus & CoRoT-7b ${ }^{b}$ & $G J^{b}$ & $55 \mathrm{CnC} \mathrm{e}^{\mathrm{b}}$ \\
\hline$T_{\text {orb }}(\mathrm{d})$ & 1.77 & 3.55 & 7.16 & 16.69 & 15.95 & $1.37^{c}$ & 0.854 & 1.58 & 0.7365 \\
\hline $2 e\left(10^{3}\right)$ & 8.2 & 18.8 & 2.6 & 14.8 & 57.6 & $9.4^{d}$ & 2 & 2 & 114 \\
\hline$\epsilon\left(10^{4}\right)$ & $1.3^{b}$ & $2^{b}$ & 0.056 & 0.042 & 1.3 & $21^{d}$ & 0.02 & 0.02 & 1.14 \\
\hline \multirow[t]{2}{*}{$R(\mathrm{~km})$} & 1,822 & 1,561 & 2,631 & 2,410 & 2,576 & $252.1^{d}$ & 10,703 & 17,062 & 10,385 \\
\hline & core & & core & & & & (possible) core & (possible) core & (possible) core \\
\hline$R_{\text {out }} / R$ & 0.52 & & 0.27 & & & & $1 / 3$ & $1 / 3$ & $1 / 3$ \\
\hline$\beta\left(10^{4}\right)$ & 60 & & 3.7 & & & & 70 & 60 & 50 \\
\hline$\chi$ & 0 & & 0 & & & & 0 & 0 & 0 \\
\hline \multirow[t]{2}{*}{$E\left(10^{14}\right)$} & 2.7 & & 20 & & & & 0.094 & 0.068 & 0.086 \\
\hline & & ocean & ocean & ocean & ocean & ocean & & & \\
\hline$R_{\text {out }} / R$ & & 0.99 & 0.96 & 0.94 & 0.97 & $0.91^{\mathrm{e}}$ & & & \\
\hline$\beta\left(10^{4}\right)$ & & 9.7 & 3.7 & 0.72 & 1.2 & $50^{f}$ & & & \\
\hline$\chi$ & & 0.94 & 0.94 & 0.93 & 0.92 & $0.84^{\mathrm{d}, \mathrm{e}}$ & & & \\
\hline$E\left(10^{14}\right)$ & & 2 & 1.5 & 4.5 & 3.5 & $36^{\mathrm{d}, e, g}$ & & & \\
\hline
\end{tabular}

${ }^{a}$ Except for Enceladus, all these values are taken from Cébron et al. [2012b, and references therein]. The physical libration amplitude of the Super-Earths is assumed to be $10^{-3}$ times the optical libration amplitude $2 e$.

${ }^{b}$ Cébron et al. [2012b, and references therein].

'McKinnon [2015].

dThomas et al. [2016].

eCalculated from Beuthe et al. [2016].

fProvided by M. Beuthe, A. Rivoldini, and A. Trinh (personal communication, 2017). The value provided was obtained by a Bayesian inversion of Enceladus' gravity and shape data as detailed in Beuthe et al. [2016]. Note that contrary to the other bodies of this table, this method accounts for the nonhydrostatic deformation of the ice-ocean interface. The results of the inversion show that $|\beta|$ can span from 0 to $300 \times 10^{-4}$, with a maximum of the distribution curve at $50 \times 10^{-4}$. For $80 \%$ of the models, $|\beta|>32 \times 10^{-4}$ (M. Beuthe, A. Rivoldini, and A. Trinh, personal communication, 2017), and Enceladus' ocean is above the instability threshold determined experimentally (see Figure 12).

${ }^{g}$ Kinematic viscosity $v$ taken as $\sim 10^{-6} \mathrm{~m}^{2} / \mathrm{s}$.

\section{Acknowledgments}

D.L. acknowledges École Normale Supérieure de Lyon for funding her internship visit in Los Angeles. A.M.G. and J.M.A. gratefully acknowledge the financial support of the NSF Geophysics Program via award 1547269. M.L.B. and B.F. acknowledge support from the European Research Council (ERC) under the European Union's Horizon 2020 research and innovation program (grant agreement 681835-FLUDYCO-ERC-2015-CoG).

This work was granted access to the HPC resources of GENCI-IDRIS (project A0020407543) and of Aix-Marseille Université financed by the project Equip@Meso (ANR-10-EQPX-29-01) of the program "Investissements d'Avenir" supervised by the Agence Nationale de la Recherche. J.V. acknowledges the French Ministère de l'Enseignement Supérieur et de la Recherche for his PhD grant. This work was partially funded by the French Agence Nationale de la Recherche under grants ANR-13-JS05-0004-01 (LIPSTIC), ANR-14-CE33-0012 (MagLune), and by the 2017 TelluS program from CNRS-INSU (PNP) AO2017-1040353. To reproduce the numerical simulations, the spectral element solver Nek5000 used is becomes clearly recognizable (third snapshot) and the whole fluid becomes unstable. As observed in the full ellipsoid case by Grannan et al. [2014], the triadic resonance at $f=2.4$ involves a coupling of spin-over modes, which are characterized by a solid-body rotation around an axis perpendicular to the rotation axis [Lacaze et al., 2005]. The frequency content is surprisingly clear in this case, and we identify with good confidence the excitation of (probable) modes of frequencies $\omega=f / 2=1.2$, followed by the secondary couples of peaks $|\omega|=[0.3,0.9],|\omega|=[0.33,0.87]$, and $|\omega|=[0.58,0.62]$. Figure $C 1 c$ shows that the spatial difference is now more subtle. The same peaks are present in both spectra, but on the side of the core, the two couples $|\omega|=[0.33,0.87]$ and $|\omega|=[0.58,0.62]$ are attenuated in comparison to the others.

Another case at $f=2.4$ has been realized, with a larger core $(\chi=0.49)$ and farther from the threshold of the instability. The same succession of phases is observed, but the flow becomes more turbulent compared to the previous case. The associated spectra are then less clean, still dominated by the $\omega=1.2$ frequency, but with less evident secondary resonances, the only one identified with certainty being the couple $\omega=[0.42,0.78]$, which was not present in the first case described. This result supports the previously mentioned idea that the excited modes change according to the radius ratio of the shell.

\section{Appendix D: Physical Characteristics Used for the Stability Analysis}

Table D1 gives physical parameters of different fluid layers present in some planetary bodies. These parameters are used to calculate all relevant dimensionless numbers for each fluid layer, which are then used in Figure 12 for a comparison with the instability threshold determined experimentally.

\section{References}

Aldridge, K. D., and A. Toomre (1969), Axisymmetric inertial oscillations of a fluid in a rotating spherical container, J. Fluid Mech., 37(2), 307-323.

Arkani-Hamed, J., B. Seyed-Mahmoud, K. D. Aldridge, and R. E. Baker (2008), Tidal excitation of elliptical instability in the Martian core: Possible mechanism for generating the core dynamo, J. Geophys. Res., 113, E06003, doi:10.1029/2007JE002982. 
available online [Fischer et al., 2008]. Figure 3, Table 1, and the table in the supporting information file give the parameters needed to reproduce the experiments. Any additional experimental data, namely, high-resolution movies, are available upon request from Daphné Lemasquerier (e-mail: d.lemasquerier@gmail.com).
Aurnou, J., M. Heimpel, L. Allen, E. King, and J. Wicht (2008), Convective heat transfer and the pattern of thermal emission on the gas giants, Geophys. J. Int., 173(3), 793-801, doi:10.1111/j.1365-246X.2008.03764.x.

Backus, G., and M. Rieutord (2016), Completeness of inertial modes of an incompressible non-viscous fluid in a corotating ellipsoid, Phys. Rev. E, 95, 053116.

Barker, A. J. (2016), Non-linear tides in a homogeneous rotating planet or star: Global simulations of the elliptical instability, Mon. Not. $R$. Astron. Soc., 459(1), 939-956, doi:10.1093/mnras/stw702.

Busse, F. (2010), Mean zonal flows generated by librations of a rotating spherical cavity, J. Fluid Mech., 650, 505-512.

Beuthe, M., A. Rivoldini, and A. Trinh (2016), Enceladus's and Dione's floating ice shells supported by minimum stress isostasy, Geophys. Res. Lett., 43(19), 10,088-10,096, doi:10.1002/2016GL070650.

Calkins, M. A., J. Noir, J. D. Eldredge, and J. M. Aurnou (2010), Axisymmetric simulations of libration-driven fluid dynamics in a spherical shell geometry, Phys. Fluids, 22(8), 086602.

Cébron, D., M. Le Bars, J. Leontini, P. Maubert, and P. Le Gal (2010a), A systematic numerical study of the tidal instability in a rotating triaxial ellipsoid, Phys. Earth Planet. Inter., 182(1), 119-128.

Cébron, D., P. Maubert, and M. Le Bars (2010b), Tidal instability in a rotating and differentially heated ellipsoidal shell, Geophys. J. Int., 182(3), 1311, doi:10.1111/j.1365-246X.2010.04712.x.

Cébron, D., M. Le Bars, J. Noir, and J. M. Aurnou (2012a), Libration driven elliptical instability, Phys. Fluids, 24(6), 061703.

Cébron, D., M. Le Bars, C. Moutou, and P. Le Gal (2012b), Elliptical instability in terrestrial planets and moons, Astron. Astrophys., 539, A78.

Cébron, D., S. Vantieghem, and W. Herreman (2014), Libration-driven multipolar instabilities, J. Fluid Mech., 739, $502-543$.

Dwyer, C. A., D. J. Stevenson, and F. Nimmo (2011), A long-lived lunar dynamo driven by continuous mechanical stirring, Nature, 479(7372), $212-214$.

Favier, B., A. J. Barker, C. Baruteau, and G. I. Ogilvie (2014), Non-linear evolution of tidally forced inertial waves in rotating fluid bodies, Mon. Not. R. Astron. Soc., 439(1), 845-860.

Favier, B., A. M. Grannan, M. Le Bars, and J. M. Aurnou (2015), Generation and maintenance of bulk turbulence by libration-driven elliptical instability, Phys. Fluids, 27(6), 066601.

Fischer, P. F., F. Loth, S. Lee, S. Lee, D. Smith, and H. Bassiouny (2007), Simulation of high-Reynolds number vascular flows, Comput. Meth. Appl. Mech. Eng., 196, 3049-3060, doi:10.1016/j.cma.2006.10.015.

Fischer, P. F., J. W. Lottes, and S. G. Kerkemeier (2008), nek5000. [Available at http://nek5000.mcs.anl.gov.]

Grannan, A. M., M. Le Bars, D. Cébron, and J. M. Aurnou (2014), Experimental study of global-scale turbulence in a librating ellipsoid, Phys. Fluids, 26(12), 126601.

Grannan, A. M., B. Favier, M. Le Bars, and J. M. Aurnou (2017), Tidally forced turbulence in planetary interiors, Geophys. J. Int., 208(3), 1690, doi:10.1093/gji/ggw479.

Greenspan, H. P. (1968), The Theory of Rotating Fluids, Cambridge Univ. Press, New York.

Hecht, F., P. J. Mucha, and G. Turk (2010), Virtual rheoscopic fluids, IEEE Trans. Visual Comput. Graphics, 16(1), 147-160.

Herreman, W., M. Le Bars, and P. Le Gal (2009), On the effects of an imposed magnetic field on the elliptical instability in rotating spheroids, Phys. Fluids, 21(4), 046602.

Hide, R., and C. Titman (1967), Detached shear layers in a rotating fluid, J. Fluid Mech., 29(1), 39-60.

Hollerbach, R., and R. R. Kerswell (1995), Oscillatory internal shear layers in rotating and precessing flows, J. Fluid Mech., 298, $327-339$.

Kerswell, R. R. (1994), Tidal excitation of hydromagnetic waves and their damping in the Earth, J. Fluid Mech., 274, $219-241$.

Kerswell, R. R. (1995), On the internal shear layers spawned by the critical regions in oscillatory Ekman boundary layers, J. Fluid Mech., 298, $311-325$.

Kunnen, R. P. J., H. J. H. Clercx, and G. J. F. van Heijst (2013), The structure of sidewall boundary layers in confined rotating Rayleigh-Bènard convection, J. Fluid Mech., 727, 509-532, doi:10.1017/jfm.2013.285.

Lacaze, L., P. Le Gal, and S. Le Dizès (2004), Elliptical instability in a rotating spheroid, J. Fluid Mech., 505, 1-22.

Lacaze, L., P. Le Gal, and S. Le Dizès (2005), Elliptical instability of the flow in a rotating shell, Phys. Earth Planet. Inter., 151(3), 194-205.

Landeau, M., P. Olson, R. Deguen, and B. H. Hirsh (2016), Core merging and stratification following giant impact, Nat. Geosci., 9(10), 786-789.

Le Bars, M., M. A. Wieczorek, Ö. Karatekin, D. Cébron, and M. Laneuville (2011), An impact-driven dynamo for the early Moon, Nature, 479(7372), 215-218.

Le Bars, M., D. Cébron, and P. Le Gal (2015), Flows driven by libration, precession, and tides, Annu. Rev. Fluid Mech., 47, $163-193$.

Le Dizès, S. (2000), Three-dimensional instability of a multipolar vortex in a rotating flow, Phys. Fluids, 12(11), $2762-2774$.

Le Reun, T., B. Favier, A. J. Barker, and M. Le Bars (2017), Inertial wave turbulence driven by elliptical instability, Phys. Rev. Lett., 119, 034502, doi:10.1103/PhysRevLett.119.034502.

Lemasquerier, D., A. Grannan, B. Favier, J. Vidal, D. Cébron, J. M. Aurnou, and M. Le Bars (2016), Ellipsoidal Shell Libration: The Movie. [Available at https://www.youtube.com/watch?v=WGe-vLsm9Ho.]

Levy, M., and H. Fernando (2002), Turbulent thermal convection in a rotating stratified fluid, J. Fluid Mech., 467, $19-40$.

Liao, X., and K. Zhang (2008), On viscous decay factors for spherical inertial modes in rotating planetary fluid cores: Comparison between asymptotic and numerical analysis, Phys. Earth Planet. Inter., 169(1), 211-219.

McKinnon, W. B. (2015), Effect of Enceladus's rapid synchronous spin on interpretation of Cassini gravity, Geophys. Res. Lett., 42, 2137-2143, doi:10.1002/2015GL063384.

Messio, L., C. Morize, M. Rabaud, and F. Moisy (2008), Experimental observation using particle image velocimetry of inertial waves in a rotating fluid, Exp. Fluids, 44(4), 519-528.

Meunier, P., and T. Leweke (2003), Analysis and treatment of errors due to high velocity gradients in particle image velocimetry, Exp. Fluids, 35(5), 408-421, doi:10.1007/s00348-003-0673-2.

Noir, J., F. Hemmerlin, J. Wicht, S. M. Baca, and J. M. Aurnou (2009), An experimental and numerical study of librationally driven flow in planetary cores and subsurface oceans, Phys. Earth Planet. Inter., 173(1), 141-152.

Noir, J., M. A. Calkins, M. Lasbleis, J. Cantwell, and J. M. Aurnou (2010), Experimental study of libration-driven zonal flows in a straight cylinder, Phys. Earth Planet. Inter., 182(1), 98-106.

Noir, J., D. Cébron, M. Le Bars, A. Sauret, and J. M. Aurnou (2012), Experimental study of libration-driven zonal flows in non-axisymmetric containers, Phys. Earth Planet. Inter., 204, 1-10.

Ogilvie, G. I. (2009), Tidal dissipation in rotating fluid bodies: A simplified model, Mon. Not. R. Astron. Soc., 396(2), $794-806$.

Phillips, O. M. (1963), Energy transfer in rotating fluids by reflection of inertial waves, Phys. Fluids, 6(4), 513-520.

Proudman, I. (1956), The almost-rigid rotation of viscous fluid between concentric spheres, J. Fluid Mech., 1(5), $505-516$.

Rieutord, M. (1991), Linear theory of rotating fluids using spherical harmonics: Part II. Time-periodic flows, Geophys. Astrophys. Fluid Dyn., 59(1-4), 185-208. 
Rieutord, M., and L. Valdettaro (2010), Viscous dissipation by tidally forced inertial modes in a rotating spherical shell, J. Fluid Mech., 643, 363-394.

Rieutord, M., B. Georgeot, and L. Valdettaro (2001), Inertial waves in a rotating spherical shell: Attractors and asymptotic spectrum, J. Fluid Mech., 435, 103-144.

Sauret, A. (2012), Forcage harmonique d'écoulements en rotation: Vents zonaux, ondes inertielles et instabilités, PhD thesis, Univ. d'Aix-Marseille.

Seyed-Mahmoud, B., G. Henderson, and K. Aldridge (2000), A numerical model for elliptical instability of the Earth's fluid outer core, Phys. Earth Planet. Inter., 117(1), 51-61.

Seyed-Mahmoud, B., K. Aldridge, and G. Henderson (2004), Elliptical instability in rotating spherical fluid shells: Application to Earth's fluid core, Phys. Earth Planet. Inter., 142, 257-282, doi:10.1016/j.pepi.2004.01.001.

Stewartson, K. (1957), On almost rigid rotations, J. Fluid Mech., 3(1), 17-26.

Stewartson, K. (1966), On almost rigid rotations: Part 2, J. Fluid Mech., 26(1), 131-144.

Thomas, P. C., R. Tajeddine, M. S. Tiscareno, J. A. Burns, J. Joseph, T. J. Loredo, P. Helfenstein, and C. Porco (2016), Enceladus's measured physical libration requires a global subsurface ocean, Icarus, 264, 37-47.

Tilgner, A. (1999), Driven inertial oscillations in spherical shells, Phys. Rev. E, 59(2), 1789.

Van Hoolst, T., N. Rambaux, O. Karatekin, V. Dehant, and A. Rivoldini (2008), The librations, shape, and icy shell of Europa, Icarus, 195(1), 386-399.

Vantieghem, S. (2014), Inertial modes in a rotating triaxial ellipsoid, Proc. R. Soc. A, 470(2168), 20140093.

Vantieghem, S., D. Cébron, and J. Noir (2015), Latitudinal libration driven flows in triaxial ellipsoids, J. Fluid Mech., 771, 193-228.

Vidal, J., D. Cébron, and N. Schaeffer (2016), Diffusionless hydromagnetic modes in rotating ellipsoids: A road to weakly nonlinear models?, ArXiv e-prints.

Vidal, J., D. Cébron, and N. Schaeffer (2017), Inviscid instabilities in rotating ellipsoids on eccentric Kepler orbits. [Available at https://hal.archives-ouvertes.fr/hal-01498649.]

Wu, C.-C., and P. H. Roberts (2013), On a dynamo driven topographically by longitudinal libration, Geophys. Astrophys. Fluid Dyn., 107(1-2), $20-44$.

Yseboodt, M., A. Rivoldini, T. Van Hoolst, and M. Dumberry (2013), Influence of an inner core on the long-period forced librations of Mercury, Icarus, 226(1), 41-51.

Zhang, K. (1993), On equatorially trapped boundary inertial waves, J. Fluid Mech., 248, 203-217, doi:10.1017/S0022112093000746.

Zhang, K., X. Liao, and P. Earnshaw (2004), On inertial waves and oscillations in a rapidly rotating spheroid, J. Fluid Mech., $504,1-40$.

Zhang, K., K. H. Chan, and X. Liao (2011), On fluid motion in librating ellipsoids with moderate equatorial eccentricity, J. Fluid Mech., 673, 468-479.

Zhang, K., K. H. Chan, X. Liao, and J. M. Aurnou (2013), The non-resonant response of fluid in a rapidly rotating sphere undergoing longitudinal libration, J. Fluid Mech., 720, 212-235. 